\title{
Self-Dual Vertex Operator Superalgebras And Superconformal Field Theory
}

\author{
Thomas Creutzig*1, John F. R. Duncan ${ }^{\dagger 2}$, and Wolfgang Riedler ${ }^{\ddagger 1}$ \\ ${ }^{1}$ Department of Mathematical and Statistical Sciences, University of Alberta, \\ Edmonton, Alberta T6G 2G1, Canada. \\ ${ }^{2}$ Department of Mathematics and Computer Science, Emory University, \\ Atlanta, GA 30322, U.S.A.
}

\begin{abstract}
Recent work has related the equivariant elliptic genera of sigma models with K3 surface target to a vertex operator superalgebra that realizes moonshine for Conway's group. Motivated by this we consider conditions under which a self-dual vertex operator superalgebra may be identified with the bulk Hilbert space of a superconformal field theory. After presenting a classification result for self-dual vertex operator superalgebras with central charge up to 12 we describe several examples of close relationships with bulk superconformal field theories, including those arising from sigma models for tori and K3 surfaces.
\end{abstract}

\footnotetext{
MSC2010: 17B69, 17B81, 20C34.

*Email: creutzig@ualberta.ca

${ }^{\dagger}$ Email: john.duncan@emory.edu

${ }^{\ddagger}$ Email: riedler@ualberta.ca
} 


\section{Contents}

1 Introduction

2 Background 6

2.1 Vertex Superalgebra . . . . . . . . . . . . . . . 6

2.2 Modularity . . . . . . . . . . . . . . . . . . . . . . 9

2.3 Superconformal Algebras . . . . . . . . . . . . . . . . 10

2.4 Free Fields . . . . . . . . . . . . . . . . . . . . . 14

3 Self-Dual Vertex Operator Superalgebras 15

4 Superconformal Field Theory 18

4.1 Potential Bulk Conformal Field Theory . . . . . . . . . . . . . . . . 18

4.2 Potential Bulk Superconformal Field Theory . . . . . . . . . . . . . . 20

4.3 Superconformal structure . . . . . . . . . . . . . . . . 23

5 Examples

5.1 Type D Conformal Field Theory . . . . . . . . . . . . . 25

5.2 Type D Superconformal Field Theory . . . . . . . . . . . . . . . . . . . . . .

5.3 Type A Superconformal Field Theory . . . . . . . . . . . . 28

5.4 Gepner Type Superconformal Field Theory . . . . . . . . . . . . . . 29

References

\section{Introduction}

The elliptic genus of a complex K3 surface $X$ is a weak Jacobi form of weight zero and index one. It may be realized in the following three ways: via the chiral de Rham complex of $X$ [2, 59, 4, 5], as the $S^{1}$-equivariant $\chi_{y}$-genus of the loop space of $X$ [60, 61, 69], and as a trace on the Ramond-Ramond sector of a sigma model on $X$ [38, 21, 88]. The small $N=4$ superconformal algebra at central charge $c=6$ appears in each of these three pictures. Firstly, it is the algebra of global sections of the chiral de Rham complex [76, 75]. Secondly, the $\chi_{y}$-genus can be viewed as a virtual module for a certain vertex operator superalgebra that contains this Lie superalgebra [17, 78. Finally, it appears as a supersymmetry algebra of the string theory sigma model [41]. (We refer to [87] for a recent detailed review of these topics.)

The K3 elliptic genus and the $N=4$ superconformal algebra were absorbed into the orbit of moonshine when Eguchi-Ooguri-Tachikawa [37] suggested a relationship between the largest Mathieu group, $M_{24}$, and character contributions of the $N=4$ superconformal algebra to the K3 elliptic genus. This ignited a resurgence of interest in connections between string theory, modular forms and finite groups. Umbral 
moonshine [9, 10, 32, Thompson moonshine [58, 56] and the recently announced O'Nan moonshine [35] all belong to the quickly developing legacy of this Mathieu moonshine observation, although the connections to string theory are so far more obscure in the latter two cases. We refer to [31] for a fuller review, more references, and for comparison to the original monstrous moonshine [15, 83, 84] that appeared in the 1970s.

By now there are indications that Mathieu and monstrous moonshine are interrelated. An instance of this, and a primary motivation for the present work is [34, wherein the K3 elliptic genus apparently makes a fourth appearance: as a trace function on the moonshine module [30, 33] for Conway's group, $C_{0}$ [13, 14]. On the one hand, this Conway moonshine module - vertex operator superalgebra with $N=1$ structure - is a direct supersymmetric analogue of the monstrous moonshine module of Frenkel-Lepowsky-Meurman [44, 45, 46]. It manifests a genus zero property for $\mathrm{Co}_{0}$ [33], just as the monstrous moonshine module does for the monster [3]. On the other hand, $\mathrm{M}_{24}$ is a subgroup of $\mathrm{Co}_{0}$, and the Conway moonshine construction of the K3 elliptic genus may be twined by (most) elements of $M_{24}$. In many, but not all instances the resulting trace functions coincide with those that arise in Mathieu moonshine.

This tells us that the Conway moonshine module comes close to providing a vertex algebraic realization of the as yet elusive Mathieu moonshine module, whose structure as a representation of $M_{24}$ was conjecturally determined in [6, 49, 50, 36, and confirmed in [54]. The Conway moonshine module has been used to realize analogues of the Mathieu moonshine module for other sporadic simple groups in [7, 11]. See [79, 80, 81, 52, 82] for the development of a promising geometric approach to the problem.

The Conway moonshine module is also connected to string theory on K3 surfaces. As is explained in [34, the Conway moonshine construction of the K3 elliptic genus may also be twined, in an explicitly computable way, by any automorphism of a K3 sigma model that preserves its supersymmetry. Such automorphisms are classified in [51. It appears that the construction of [34] is (except for a small number of possible exceptions) in agreement [12] with the twined K3 elliptic genera that one expects [12] to arise from string theory. One reason this is surprising is that K3 sigma models, and in particular their automorphisms, are difficult to construct in general (cf. e.g. [71]). The Conway moonshine module seems to serve as a shortcut, to certain computations which might otherwise require the explicit construction of sigma models.

In view of these connections it is natural to ask how the Conway moonshine realization of the K3 elliptic genus is related to the three we began with above. Katz-Klemm-Vafa [67] conjectured a method for computing the Gromov-Witten invariants of a K3 surface in terms of the $\chi_{y}$-genera of its symmetric powers, and the 
generating function of these $\chi_{y}$-genera can be realized in terms of a lift of the K3 elliptic genus. So the second mentioned realization of the K3 elliptic genus, as a generalization of Hirzebruch's $\chi_{y}$-genus, suggests a connection between Conway moonshine and enumerative geometry. This perspective is developed in [8], where equivariant counterparts to the conjecture of Katz-Klemm-Vafa are formulated, which explicitly describe equivariant versions of Gromov-Witten invariants of K3 surfaces. The conjecture of Katz-Klemm-Vafa was proved recently by Pandharipande-Thomas [72]. Katz-Klemm-Pandharipande [66] have extended the the conjecture of Katz-KlemmVafa to refined Gopakumar-Vafa invariants. Conjectural descriptions of equivariant refined Gopakumar-Vafa invariants of K3 surfaces are also formulated in [8].

In this work we develop the relationship between Conway moonshine and the third mentioned realization, in terms of K3 sigma models. We do this by formalizing a new relationship between vertex algebra and conformal field theory, and by realizing the Conway moonshine module, and other vertex operator superalgebras, in examples.

Traditionally, vertex operator algebras satisfying suitable conditions are considered to define "chiral halves" of conformal field theories. More specifically, the bulk Hilbert space of a conformal field theory may be regarded as (a completion of) a suitable sum of modules for a tensor product of vertex operator algebras (cf. [63, 48, 87]). The alternative viewpoint we pursue here develops from the observation that a bulk Hilbert space of a conformal field theory, taken as a whole, resembles a self-dual vertex operator algebra. We explain this observation more fully in $\$ 4.1$. It motivates our

Main Question: Can a self-dual vertex operator algebra be identified with a bulk conformal field theory in some sense?

We answer this question positively by formulating the notion of potential (bulk) conformal field theory (cf. Definition 4.1) and by identifying self-dual vertex operator algebras as examples (cf. Propositions 5.1 and 5.3). In fact, we formulate supersymmetric counterparts to potential conformal field theories as well (cf. Definitions 4.3 and 4.6), and find more examples amongst self-dual vertex operator superalgebras (cf. Theorems 5.6, 5.8 and 5.9). To support the analysis we also present a classification result (Theorem 3.1) for self-dual vertex operator superalgebras with central charge up to 12 .

Equipped with the notion of potential bulk superconformal field theory we relate the Conway moonshine module to four superconformal field theories in 95 , One of these is the superconformal field theory underlying the tetrahedral K3 sigma model (cf. \$5.3), which was analyzed in detail by Gaberdiel-Taormina-Volpato-Wendland [53] (see also [82]). Another is the Gepner model $(1)^{6}$ (cf. \$5.4), and it is clear that there are further interesting examples waiting to be considered, that may shed more light on the role of Conway moonshine in K3 string theory. 
An implication of our analysis is that there should be self-dual vertex operator superalgebras besides the Conway moonshine module that have analogous relationships to other string theory compacitifcations. In $\$ 5.2$ we identify a self-dual vertex operator superalgebra - the $N=1$ vertex operator superalgebra naturally attached to the $E_{8}$ lattice - which realizes the bulk superconformal field theory underlying a sigma model with a 4-torus as target (cf. Theorem [5.6). Volpato [86] has shown that the supersymmetry preserving automorphism groups of 4-torus sigma models are subgroups of the Weyl group of the $E_{8}$ lattice. In light of these results it seems likely that the $E_{8}$ vertex operator superalgebra that appears in $\$ 5.3$ can serve as a counterpart to the Conway moonshine module for nonlinear sigma models on 4-dimensional tori.

It is interesting to compare the approach presented here to recent work [82] of Taormina-Wendland. In loc. cit. the relationship between superconformal field theory and vertex operator superalgebra is also reconsidered, but the starting point is a fully fledged superconformal field theory. A notion of reflection is introduced which, in special circumstances, produces a vertex operator superalgebra. The superconformal field theory underlying the tetrahedral K3 sigma model is considered in detail, and it is shown that the Conway moonshine module arises when reflection is applied in this case. In this way Taormina-Wendland independently obtain results equivalent to those we present in $\$ 5.3$. Our notion of potential superconformal field theory serves to answer the question of what reflection produces from a superconformal field theory in general, except that a reflected superconformal field theory comes equipped with extra structure, on account of the richness of the superconformal field theory axioms. For example, the reflected tetrahedral K3 theory recovers the vertex operator superalgebra structure on the Conway moonshine module, but also furnishes an intertwining operator algebra structure on the direct sum of itself with its unique irreducible canonically twisted module (cf. $\S 4$ of [82]). Moving forward, we can expect that Taormina-Wendland reflection will play a key role in further elucidating the relationships between superconformal field theories, potential superconformal field theories and vertex operator superalgebras.

We now describe the structure of the article. We present background material in 92 . We explain our conventions on vertex operator superalgebras in 2.1 , and we review some modularity results for vertex operator superalgebras in 2.2 . We recall the small $N=4$ superconformal algebra in $\$ 2.3$, and describe an explicit construction at $c=6$ in 2.4 . In $\$ 3$ we establish our classification result for selfdual vertex operator superalgebras with central charge at most 12 . Then in $\$ 4$ we discuss the new relationship between vertex algebra and conformal field theory that motivates this work, and explain our approach to answering the Main Question. We begin with conformal field theory in 4.1 discuss superconformal field theory in 4.2 , and consider superconformal field theories with superconformal structure in 
4.3. We present examples of bulk superconformal field theory interpretations of self-dual vertex operator superalgebras in $\$ 5$. We begin with the diagonal conformal field theories associated to type $D$ lattice vertex operator algebras in $₫ 5.1$, and then discuss super analogues of these in $\$ 5.2$. We discuss the superconformal field theory underlying the tetrahedral K3 sigma model in \$5.3, and discuss the relationship between the Conway moonshine module and the Gepner model $(1)^{6}$ in $\$ 5.4$.

\section{Background}

\section{$2.1 \quad$ Vertex Superalgebra}

We assume some familiarity with the basics of vertex (operator) superalgebra theory. Good references for this include [42, 46, 64, 70].

We adopt the convention, common in physical settings, of writing $(-1)^{F}$ for the canonical involution on a superspace $W=W^{\text {even }} \oplus W^{\text {odd }}$, so that $\left.(-1)^{F}\right|_{W^{\text {even }}}=I$ and $\left.(-1)^{F}\right|_{W_{\text {odd }}}=-I$. We write $Y(a, z)=\sum a_{(k)} z^{-k-1}$ for the vertex operator attached to an element $a$ in a vertex superalgebra $W$. A vertex superalgebra $W$ is called $C_{2}$-cofinite if $W / C_{2}(W)$ is finite-dimensional, where $C_{2}(W):=\left\{a_{(-2)} b \mid a, b \in W\right\}$. Following [24] we say that a vertex operator superalgebra $W$ is of CFT type if the $L_{0}$-grading $W=\bigoplus_{n \in \frac{1}{2} \mathbb{Z}} W_{n}$ is bounded below by 0 , and if $W_{0}$ is spanned by the vacuum vector. We assume that $W^{\text {even }}=\bigoplus_{n \in \mathbb{Z}} W_{n}$ and $W^{\text {odd }}=\bigoplus_{n \in \mathbb{Z}+\frac{1}{2}} W_{n}$. A vertex operator superalgebra that is $C_{2}$-cofinite and of CFT type is nice (schön) in the sense of 62 .

Say that a vertex operator algebra is rational if all of its admissible modules are completely reducible. We refer to [22] for the definition of admissible module. It is proven in loc. cit. that a rational vertex operator algebra has finitely many irreducible admissible modules up to equivalence. We say that a vertex operator superalgebra is rational if its even sub vertex operator algebra is rational. We will apply results from [28] in what follows, so we should note that our notion of rationality for a vertex operator superalgebra is stronger than that which appears there. A vertex operator superalgebra that is rational in our sense is both rational and $(-1)^{F}$-rational in the sense of loc. cit. The equivalence of the two notions of rationality is proven in 57] under an assumption on fusion products of canonically twisted modules.

We say that a vertex operator superalgebra $W$ is self-dual if $W$ is rational (in our sense), irreducible as a $W$-module, and if $W$ is the only irreducible admissible $W$ module up to isomorphism. Note that the term self-dual is sometimes used differently elsewhere in the literature, to refer to the situation in which $W$ is isomorphic to its contragredient as a $W$-module.

According to Theorem 8.7 of [28] a self-dual $C_{2}$-cofinite vertex operator superalgebra $W$ has a unique (up to isomorphism) irreducible $(-1)^{F}$-stable canonically twisted module. We denote it $W_{\mathrm{tw}}$. The $(-1)^{F}$-stable condition on a canonically 
twisted module $M$ for $W$ is equivalent to the requirement of a superspace structure $M=M^{\text {even }} \oplus M^{\text {odd }}$ that is compatible with the superspace structure on $W$, so that elements of $W^{\text {even }}$ and $W^{\text {odd }}$ induce even and odd transformations of $M$, respectively. Modules that are not $(-1)^{F}$-stable will not arise in this work so we henceforth assume the existence of a compatible superspace structure to be a part of the definition of untwisted or canonically twisted module for a vertex operator superalgebra. However, we will not require morphisms of modules to preserve a particular superspace structure. So for example, if $W$ is a vertex operator superalgebra and $\Pi$ is the parity change functor on superspaces then $W$ and $\Pi W$ are not isomorphic as superspaces, but we do regard them as isomorphic $W$-modules.

Write $V_{L}$ for the vertex superalgebra attached to an integral lattice $L$, which is naturally a vertex operator superalgebra if $L$ is positive definite. Write $F(n)$ for the vertex operator superalgebra of $n$ free fermions. According to the boson-fermion correspondence [43, 25] the vertex operator superalgebra attached to $\mathbb{Z}^{n}$ is isomorphic to $F(2 n)$. So the even sub vertex operator algebra $F(2 n)^{\text {even }}<F(2 n)$ is isomorphic to the lattice vertex operator algebra attached to the type $D$ lattice

$$
D_{n}:=\left\{\left(x_{1}, \ldots, x_{n}\right) \in \mathbb{Z}^{n} \mid x_{1}+\cdots+x_{n}=0 \bmod 2\right\} .
$$

The discriminant group of $D_{n}$ is $D_{n}^{*} / D_{n} \simeq \mathbb{Z} / 2 \mathbb{Z} \times \mathbb{Z} / 2 \mathbb{Z}$, and we label coset representatives as follows.

$$
\begin{aligned}
& {[0]:=(0, \ldots, 0,0), \quad[1]:=\frac{1}{2}(1, \ldots, 1,1),} \\
& {[2]:=(0, \ldots, 0,1), \quad[3]:=\frac{1}{2}(1, \ldots, 1,-1) .}
\end{aligned}
$$

Set $D_{n}^{+}:=D_{n} \cup D_{n}+[1]$. Then $D_{n}^{+}$is a self-dual integral lattice - the rank $n$ spin lattice-whenever $n=0 \bmod 4$. It is even if $n=0 \bmod 8$. We have $D_{4}^{+} \cong \mathbb{Z}^{4}$ and $D_{8}^{+} \cong E_{8}$, and $D_{12}^{+}$is the unique self-dual integral lattice of rank 12 such that $\lambda \cdot \lambda \leq 1$ implies $\lambda=0$. The lattice vertex operator superalgebras attached to $D_{n}$ and $D_{4 n}^{+}$ will play a prominent role later on.

Set $A_{1}=\sqrt{2} \mathbb{Z}$. We will make use of the fact that $D_{2 n}$ admits $A_{1}^{2 n}$ as a sub lattice. Explicitly, denoting $e_{1}:=(1,0, \ldots 0), e_{2}:=(0,1, \ldots, 0), \& c$., we may take the first copy of $A_{1}$ to be generated by $e_{1}+e_{n+1}$, the second copy to be generated by $e_{1}-e_{n+1}$, the third copy to be generated by $e_{2}+e_{n+2}$, \&c. In the case that $n=2$ this embedding is actually an isomorphism, $D_{2} \cong A_{1} \oplus A_{1}$. More generally, $D_{2 n} / A_{1}^{2 n}$ embeds in the discriminant group of $A_{1}^{2 n}$, which is naturally isomorphic to $(\mathbb{Z} / 2 \mathbb{Z})^{2 n} \cong \mathbb{F}_{2}^{2 n}$. As such, it is natural to use binary codewords of length $2 n$ to label cosets of $A_{1}^{2 n}$ in its dual. Given such a codeword $C \in \mathbb{F}_{2}^{2 n}$, define wt $(C)$ - the weight of $C$ - to be the number of non-zero entries of $C$. Define a binary code $\mathcal{D}_{2 n}<\mathbb{F}_{2}^{2 n}$ 
by setting

$$
\mathcal{D}_{2 n}:=\left\{C=\left(c_{1}, \ldots, c_{2 n}\right) \mid c_{i}=c_{n+i} \text { for } 1 \leq i \leq n, \operatorname{wt}(C)=0 \bmod 4\right\}
$$

We will abuse notation somewhat by also using $[i]$ to denote the following length $2 n$ codewords,

$$
[0]:=\left(0^{2 n}\right), \quad[1]:=\left(1^{n} 0^{n}\right), \quad[2]:=\left(0^{n-1} 10^{n-1} 1\right), \quad[3]:=\left(1^{n-1} 0^{n} 1\right) .
$$

The next result may be checked directly, and smooths out any conflict between (2.2) and (2.4).

Lemma 2.1. With the above conventions, the image of $\left(D_{2 n}+[i]\right) / A_{1}^{2 n}$ in $\mathbb{F}_{2}^{2 n}$ is $\mathcal{D}_{2 n}+[i]$ for $i \in\{0,1,2,3\}$.

The above discussion shows, in particular, that $A_{1}^{12} \cong \sqrt{2} \mathbb{Z}^{12}$ embeds in $D_{12}^{+}$. In $\$ 5.4$ we will make use of the fact that $\sqrt{3} \mathbb{Z}^{12}$ also embeds in $D_{12}^{+}$. To see this recall that the (extended) ternary Golay code is a linear sub space $\mathcal{G}<\mathbb{F}_{3}^{12}$ of dimension 6 such that if

$$
C \cdot D:=\sum_{i} c_{i} d_{i}
$$

for $C=\left(c_{1}, \ldots, c_{12}\right)$ and $D=\left(d_{1}, \ldots, d_{12}\right)$ then $C \cdot D=0$ when $C, D \in \mathcal{G}$, and no non-zero codeword $C \in \mathcal{G}$ has less than six non-zero entries. These properties determine $\mathcal{G}$ uniquely, up to permutations of coordinates, and multiplications of coordinates by \pm 1 (cf. e.g. [16]).

We will denote the elements of $\mathbb{F}_{3}$ by $\{0,+,-\}$ when convenient. To obtain an embedding of $\sqrt{3} \mathbb{Z}^{12}$ in $D_{12}^{+}$fix a copy $\mathcal{G}$ of the ternary Golay code in $\mathbb{F}_{3}^{12}$. Multiplying some components by -1 if necessary we may assume that $\left(+{ }^{12}\right) \in \mathcal{G}$. Then there are exactly 11 code words $C^{i}=\left(c_{1}^{i}, \ldots, c_{12}^{i}\right) \in \mathcal{G}$ such that the first entry of $C^{i}$ is +1 , five further entries are +1 , and the remaining six entries are -1 . Set $C^{12}=\left({ }^{12}\right)$ and define $\lambda^{i}:=\left(\lambda_{1}^{i}, \ldots, \lambda_{12}^{i}\right)$ for $1 \leq i \leq 12$ by setting $\lambda_{j}^{i}= \pm \frac{1}{2}$ when $c_{j}^{i}= \pm 1$. Then the $\lambda^{i}$ all belong to $D_{12}^{+}$, and satisfy $\lambda^{i} \cdot \lambda^{j}=3 \delta_{i j}$. So the $\lambda^{i}$ constructed in this way generate a sub lattice of $D_{12}^{+}$isomorphic to $\sqrt{3} \mathbb{Z}^{12}$.

The discriminant group of $\sqrt{3} \mathbb{Z}^{12}$ is $\mathbb{F}_{3}^{12}$, so it is natural to consider the image of $D_{12}^{+} / \sqrt{3} \mathbb{Z}^{12}$ in $\mathbb{F}_{3}^{12}$. Denote it $\mathcal{G}_{12}^{+}$. Since $D_{12}^{+}$is a self-dual lattice, $\mathcal{G}_{12}^{+}$is a linear subspace such that $C \cdot D=0$ for $C, D \in \mathcal{G}_{12}^{+}$. From the fact that $\lambda \in D_{12}^{+}$can only satisfy $\lambda \cdot \lambda \leq 1$ if $\lambda=0$ we obtain that $\mathcal{G}_{12}^{+}$has no non-zero words with fewer than six non-zero entries. Applying the uniqueness of the ternary Golay code we obtain the following result.

Lemma 2.2. The image of $D_{12}^{+} / \sqrt{3} \mathbb{Z}^{12}$ in $\mathbb{F}_{3}^{12}$ is a copy of the ternary Golay code. 


\subsection{Modularity}

We now review some results on modularity for vertex operator superalgebras.

Zhu proved [89] that certain trace functions on irreducible modules for suitable vertex operator algebras span representations of the modular group $S L_{2}(\mathbb{Z})$. More general modularity results that incorporate twisted modules have been obtained by Dong-Li-Mason [23], Dong-Zhao [28, 29], and Van Ekeren [85]. We will use the extension of [89, 23] to vertex operator superalgebras established in [28].

To describe the relevant results let $W=\bigoplus_{n \in \frac{1}{2} \mathbb{Z}} W_{n}$ be a rational $C_{2}$-cofinite vertex operator superalgebra of CFT type. Let $I_{0}$ be an index set for the isomorphism classes of irreducible $W$-modules, let $I_{1}$ be an index set for the isomorphism classes of irreducible canonically twisted $W$-modules, and set $I:=I_{0} \cup I_{1}$. Write $M_{i}$ for a representative (untwisted or canonically twisted) $W$-module corresponding to $i \in I$, and choose a compatible superspace structure $M_{i}=M_{i}^{\text {even }} \oplus M_{i}^{\text {odd }}$ for each $i \in I$. For $M$ an untwisted or canonically twisted $W$-module define vertex operators on the torus $Y[a, z]: M \rightarrow M((z))$ for $a \in W$ by requiring that $Y[a, z]=Y\left(a, e^{z}-1\right) e^{n z}$ when $a \in W_{n}$, and define $a_{[n]} \in \operatorname{End}(M)$ by requiring $Y[a, z]=\sum_{k} a_{[n]} z^{-n-1}$. Also, write $W=\bigoplus_{n \in \frac{1}{2} \mathbb{Z}} W_{[n]}$ for the eigenspace decomposition of $W$ with respect to the action of $\tilde{\omega}_{[1]}$, where $\tilde{\omega}:=\omega-\frac{c}{24} \mathbf{v}$, and $\omega$ and $\mathbf{v}$ are the Virasoro and vacuum elements of $W$, respectively.

Theorem 2.3 ([28]). Suppose that $W$ is a rational $C_{2}$-cofinite vertex operator superalgebra with central charge $c$. Then with $I$ and $\left\{M_{i}\right\}_{i \in I}$ as above there are maps $\rho_{i j}: S L_{2}(\mathbb{Z}) \rightarrow \mathbb{C}$ for $i, j \in I$ such that if $v \in W_{[n]}$ for some $n \in \frac{1}{2} \mathbb{Z}$ then

$$
\left.\operatorname{tr}_{M_{i}}\left(o(v)(-1)^{\ell F} q^{L_{0}-\frac{c}{24}}\right)\right|_{n}\left(\begin{array}{ll}
a & b \\
c & d
\end{array}\right)=\sum_{j \in I_{\tilde{k}}} \rho_{i j}\left(\begin{array}{ll}
a & b \\
c & d
\end{array}\right) \operatorname{tr}_{M_{j}}\left(o(v)(-1)^{\tilde{\ell} F} q^{L_{0}-\frac{c}{24}}\right)
$$

for $\ell \in \mathbb{Z} / 2 \mathbb{Z}$ and $\left(\begin{array}{ll}a & b \\ c & d\end{array}\right) \in S L_{2}(\mathbb{Z})$, where $\tilde{k}=1+a(k+1)+c(\ell+1) \bmod 2$ and $\tilde{\ell}=1+b(k+1)+d(\ell+1) \bmod 2$ when $i \in I_{k}$.

In (2.6) we utilize the usual slash notation from modular forms, setting

$$
\left(\left.f\right|_{n}\left(\begin{array}{ll}
a & b \\
c & d
\end{array}\right)\right)(\tau):=f\left(\frac{a \tau+b}{c \tau+d}\right) \frac{1}{(c \tau+d)^{n}}
$$

for a holomorphic function $f: \mathbb{H} \rightarrow \mathbb{C}$ and $\left(\begin{array}{ll}a & b \\ c & d\end{array}\right) \in S L_{2}(\mathbb{Z})$. If $W$ is a rational $C_{2^{-}}$ cofinite vertex operator algebra then we can apply Theorem 2.3 to $W$ by regarding it as a vertex operator superalgebra with trivial odd part. Then $I_{0}=I_{1}$ and we recover a specialization of Zhu's results [89] for $W$ by taking $k=\ell=1$ in (2.6).

Resume the assumption that $W$ is a rational $C_{2}$-cofinite vertex operator superalgebra of CFT type. Define the characters of an untwisted or canonically twisted 
$W$-module $M$ by setting

$$
\operatorname{ch}^{ \pm}[M](\tau):=\operatorname{tr}_{M}\left(( \pm 1)^{F} q^{L_{0}-\frac{c}{24}}\right) .
$$

Then taking $v$ to be the vacuum in Theorem 2.3 we obtain

$$
\operatorname{ch}^{\epsilon}\left[M_{i}\right]\left(\frac{a \tau+b}{c \tau+d}\right)=\sum_{j \in I_{\tilde{k}}} \rho_{i j}(\gamma) \operatorname{ch}^{\tilde{\epsilon}}\left[M_{j}\right](\tau),
$$

for $\left(\begin{array}{ll}a & b \\ c & d\end{array}\right) \in S L_{2}(\mathbb{Z})$, where $\epsilon=(-1)^{\ell}$ and $\tilde{\epsilon}=(-1)^{\tilde{\ell}}$.

In this work we will be especially interested in the situation in which a superspace $M$ is a module for a tensor product $V^{\prime} \otimes V^{\prime \prime}$ of vertex operator superalgebras, and is thus equipped with two commuting actions of the Virasoro algebra. We write $L_{n}^{\prime}$ and $L_{n}^{\prime \prime}$ for the Virasoro operators corresponding to $V^{\prime}$ and $V^{\prime \prime}$, respectively, and write $c^{\prime}$ and $c^{\prime \prime}$ for the corresponding central charges. Then it is natural to consider the refined characters

$$
\widehat{\operatorname{ch}}^{ \pm}[M]\left(\tau^{\prime}, \tau^{\prime \prime}\right):=\operatorname{tr}_{M}\left(( \pm 1)^{F} q^{\prime L_{0}^{\prime}-\frac{c^{\prime}}{24}} q^{\prime \prime L_{0}^{\prime \prime}-\frac{c^{\prime \prime}}{24}}\right)
$$

where $q^{\prime}:=e^{2 \pi i \tau^{\prime}}$ and $q^{\prime \prime}:=e^{2 \pi i \tau^{\prime \prime}}$. Krauel-Miyamoto 68, have shown how Zhu's theory 89] extends so as to yield a modularity result for such refined characters in the vertex operator algebra case. By replacing Zhu's results with the appropriate vertex operator superalgebra counterparts from [28] in the proof of Theorem 1 in [68. we readily obtain a direct analogue for vertex operator superalgebras. Here we require the following special case of this.

Theorem 2.4 ([28, 68]). Let $W$ be a rational $C_{2}$-cofinite vertex operator algebra and suppose that $\omega=\omega^{\prime}+\omega^{\prime \prime}$ where $\omega$ is the conformal vector of $W$, and $\omega^{\prime}$ and $\omega^{\prime \prime}$ generate commuting representations of the Virasoro algebra. Then with $I,\left\{M_{i}\right\}_{i \in I}$ and $\rho_{i j}(\gamma)$ as in (2.6) we have

$$
\widehat{\operatorname{ch}}^{\epsilon}\left[M_{i}\right]\left(\frac{a \tau^{\prime}+b}{c \tau^{\prime}+d}, \frac{a \tau^{\prime \prime}+b}{c \tau^{\prime \prime}+d}\right)=\sum_{j \in I_{\tilde{k}}} \rho_{i j}(\gamma) \widehat{c h}^{\tilde{\epsilon}}\left[M_{j}\right]\left(\tau^{\prime}, \tau^{\prime \prime}\right)
$$

for $\left(\begin{array}{ll}a & b \\ c & d\end{array}\right) \in S L_{2}(\mathbb{Z})$. Here $\tilde{k}$ and $\tilde{\ell}$ are as in (2.6) , for $\epsilon=(-1)^{\ell}$ and $\tilde{\epsilon}=(-1)^{\tilde{\ell}}$.

\subsection{Superconformal Algebras}

In this section we recall some properties of the small $N=4$ superconformal algebra from [1, 39, 40, 41]. It is strongly generated by a Virasoro field $T$ of dimension 2, four odd fields $G^{a}(a=0,1,2,3)$ of dimension $\frac{3}{2}$, and three even fields $J^{i}(i=1,2,3)$ 
of dimension 1. Define

$$
\alpha_{a, b}^{i}:=\frac{1}{2}\left(\delta_{a, i} \delta_{b, 0}-\delta_{b, i} \delta_{a, 0}\right)+\frac{1}{2} \epsilon_{i a b}
$$

where $\epsilon_{i j k}$ is totally antisymmetric for $i, j, k \in\{1,2,3\}$, normalized so that $\epsilon_{123}=1$, and defined to be zero if one of the indices is zero. Also let $k$ be a positive integer and set $c=6 k$. The operator product algebra in a representation with central charge $c$ is then

$$
\begin{aligned}
T(z) T(w) & \sim \frac{\frac{1}{2} c}{(z-w)^{4}}+\frac{2 T(w)}{(z-w)^{2}}+\frac{\partial_{w} T(w)}{(z-w)}, \\
T(z) G^{a}(w) & \sim \frac{\frac{3}{2} G^{a}(w)}{(z-w)^{2}}+\frac{\partial_{w} G^{a}(w)}{(z-w)}, \\
T(z) J^{i}(w) & \sim \frac{J^{i}(w)}{(z-w)^{2}}+\frac{\partial_{w} J^{i}(w)}{(z-w)}, \\
G^{a}(z) G^{b}(w) & \sim \frac{\frac{2}{3} c \delta_{a, b}}{(z-w)^{3}}-\frac{\sum_{i=1}^{3} 8 \alpha_{a, b}^{i} J^{i}(w)}{(z-w)^{2}}+\frac{2 \delta_{a, b} T(w)-\sum_{i=1}^{3} 4 \alpha_{a, b}^{i} \partial_{w} J^{i}(w)}{(z-w)}, \\
J^{i}(z) G^{a}(w) & \sim \frac{\sum_{b=0}^{3} 4 \alpha_{a, b}^{i} G^{b}(w)}{(z-w)}, \\
J^{i}(z) J^{j}(w) & \sim \frac{-\frac{1}{2} k \delta_{i, j}}{(z-w)^{2}}+\frac{\sum_{k=1}^{3} \epsilon_{i j k} J^{k}(w)}{(z-w)} .
\end{aligned}
$$

Equivalently, we have the following commutation relations of modes, where $m, n \in \mathbb{Z}$ and $r, s \in \mathbb{Z}+\frac{1}{2}$.

$$
\begin{aligned}
{\left[L_{m}, L_{n}\right] } & =(m-n) L_{m+n}=\frac{c}{12}\left(M^{3}-m\right) \delta_{m+n, 0}, \\
{\left[L_{m}, G_{r}^{a}\right] } & =\left(\frac{m}{2}-r\right) G_{m+r}^{a} \\
{\left[L_{m}, J_{n}^{i}\right] } & =-n J_{m+n}^{i}, \\
\left\{G_{r}^{a}, G_{s}^{b}\right\} & =2 \delta_{a, b} L_{r+s}-\sum_{i=1}^{3} 4(r-s) \alpha_{a, b}^{i} J_{r+s}^{i}+\frac{c}{3}\left(r^{2}-\frac{1}{4}\right) \delta_{a, b} \delta_{r+s, 0}, \\
{\left[J_{m}^{i}, G_{r}^{a}\right] } & =\sum_{b=0}^{3} \alpha_{a, b}^{i} G_{m+r}^{b}, \\
{\left[J_{m}^{i}, J_{n}^{j}\right] } & =\sum_{k=1}^{3} \epsilon_{i j k} J_{m+n}^{k}-m \frac{k}{2} \delta_{i, j} \delta_{m+n, 0} .
\end{aligned}
$$

Note that the currents $J^{i}(z)$ represent the affine Lie algebra of $\mathfrak{s l}_{2}$ at level $k$, and $k$ is a positive integer in any unitary representation. Often one uses another basis for 
these; namely

$$
J(z):=-2 i J^{1}(z), \quad J^{+}(z):=J^{2}(z)-i J^{3}(z), \quad J^{-}(z):=-J^{2}(z)-i J^{3}(z) .
$$

Also for the odd currents there is another useful basis:

$$
\begin{array}{ll}
G^{-, 1}(z):=G^{0}(z)-i G^{1}(z), & G^{+, 1}(z):=-G^{2}(z)+i G^{3}(z), \\
G^{-, 2}(z):=G^{2}(z)+i G^{3}(z), & G^{+, 2}(z):=-G^{0}(z)+i G^{1}(z),
\end{array}
$$

which yields

$$
\left[J_{m}^{ \pm}, G_{r}^{\mp, x}\right]=G_{m+r}^{ \pm, x}, \quad\left[J_{m}, G_{r}^{ \pm, x}\right]= \pm G_{m+r}^{ \pm, x}
$$

for $x \in\{1,2\}$.

The mode algebra of the affine Lie algebra of $\mathfrak{s l}_{2}$ at level $k$ has a family of automorphisms, called spectral flow. They are induced from affine Weyl translations so they are parameterized by the translation lattice which is isomorphic to $\mathbb{Z}$. For $\ell \in \mathbb{Z}$ the corresponding action is denoted $\sigma^{\ell}$, and satisfies

$$
\begin{aligned}
\sigma^{\ell}\left(J_{n}^{ \pm}\right) & =J_{n \mp \ell}^{ \pm}, \\
\sigma^{\ell}\left(J_{n}\right) & =J_{n}-\delta_{n, 0} \ell k, \\
\sigma^{\ell}\left(L_{n}\right) & =L_{n}+\delta_{n, 0}\left(\frac{\ell}{2} J_{0}+\frac{\ell^{2} k}{4}\right) .
\end{aligned}
$$

We may consider twisted modules as in (2.10) of [20]. That is, given a module $M$ for the affine Lie algebra of $\mathfrak{s l}_{2}$ at level $k$ let $\sigma_{\ell}^{*}$ be the unique invertible linear transformation of $M$ such that the action of the mode algebra satisfies

$$
X \sigma_{\ell}^{*}(v)=\sigma_{\ell}^{*}\left(\sigma^{-\ell}(X) v\right)
$$

This $\sigma^{\ell}$-twisting of $M$ is isomorphic to $M$ as a module for the mode algebra, but since the grading is changed they are in general not isomorphic as modules for the vertex operator algebra $L_{k}\left(\mathfrak{s l}_{2}\right)$ attached to $\mathfrak{s l}_{2}$ at level $k$. Identification of the twisted modules can often be achieved via characters.

So we define a character of $M$ by setting

$$
\operatorname{ch}[M](y, u, \tau):=\operatorname{tr}_{M}\left(y^{k} z^{J_{0}} q^{L_{0}-\frac{c}{24}}\right)
$$

where $z=e^{2 \pi i u}$ and $q=e^{2 \pi i \tau}$. Then from the action of $\sigma^{\ell}$ we see that

$$
\operatorname{ch}\left[\sigma_{\ell}^{*}(M)\right](y, z, q)=\operatorname{ch}[M]\left(y z^{\ell} q^{\frac{1}{4} \ell^{2}}, z q^{\frac{1}{2} \ell}, q\right) .
$$


If $k$ is a positive integer and $M$ is an irreducible integrable highest-weight module of level $k$, then the character of $M$ is a component of a vector-valued Jacobi form and the twisted module can be seen to be isomorphic to the original one if $\ell$ is even. If $\ell$ is odd it maps the irreducible integrable highest-weight module with highest weight $j$ (corresponding to the $j+1$ dimensional representation of $\mathfrak{s l}_{2}$ ) to the one with highest weight $k-j$. Thus it corresponds to fusion with the order two simple current module.

We now consider a vertex operator superalgebra $V$ of central charge $c=6 k$ that contains a commuting pair of sub vertex operator algebras $L_{k}\left(\mathfrak{s l}_{2}\right)$ and $U$, where the Virasoro elements of $V$ and $L_{k}\left(\mathfrak{s l}_{2}\right) \otimes U$ coincide. We require $U$ to be rational, and suppose that $V$ is of the form

$$
V=\bigoplus_{i} L_{k}\left(\lambda_{i}\right) \otimes U_{i}
$$

where $L_{k}\left(\lambda_{i}\right)$ is the irreducible $L_{k}\left(\mathfrak{s l}_{2}\right)$-module with highest weight $\lambda_{i}$, and $U_{i}$ is an irreducible $U$-module. Further we require that $V$ contain strong generators for the small $N=4$ superconformal algebra at $c=6 k$. In this situation the parity of elements in $V$ is given by $(-1)^{J_{0}}$ where $J_{0}$ is as in (2.15), (2.18). The spectral flow automorphism $\sigma:=\sigma^{1}$ extends naturally to $V$ in such a way that

$$
\sigma^{*}(V)=\bigoplus_{i}\left(L_{k}\left(\lambda_{i}\right) \bigotimes_{L_{k}\left(\mathfrak{s} \mathfrak{l}_{2}\right)} L_{k}\left(\lambda_{*}\right)\right) \otimes U_{i}
$$

as a $L_{k}\left(\mathfrak{s l}_{2}\right) \otimes U$-module, where $L_{k}\left(\lambda_{*}\right)$ denotes the order two simple current of $L_{k}\left(\mathfrak{s l}_{2}\right)$, and $\nabla_{V}$ is the fusion product for modules over $V$.

Under spectral flow we have $\sigma^{-1}\left(L_{0}\right)=L_{0}+\frac{1}{2} J_{0}+\frac{c}{24}$, so the conformal weight of the modes $G_{r}^{ \pm, x}$ becomes $r \pm \frac{1}{2}$ as endomorphisms on twisted modules. Especially, the $G_{\mp \frac{1}{2}}^{ \pm, x}$ act with conformal dimension zero, and thus induce grading-preserving maps from the even part to the odd part of the twisted module. We compute

$$
\begin{aligned}
\left(G_{-\frac{1}{2}}^{+, 1}+G_{\frac{1}{2}}^{-, 2}\right)^{2} & =\frac{1}{2}\left\{G_{-\frac{1}{2}}^{+, 1}+G_{\frac{1}{2}}^{-, 2}, G_{-\frac{1}{2}}^{+, 1}+G_{\frac{1}{2}}^{-, 2}\right\} \\
& =-2 L_{0}+J_{0} \\
& =2 \sigma^{-1}\left(L_{0}-\frac{c}{24}\right) .
\end{aligned}
$$

This shows that $G_{-\frac{1}{2}}^{+, 1}+G_{\frac{1}{2}}^{-, 2}$ is an invertible map from the even conformal grade $n$ subspace of $\sigma^{*}(M)$ to the odd one as long as $n-\frac{c}{24}$ does not vanish. We summarize this as follows.

Lemma 2.5. If $M$ is a $V$-module and $\sigma^{*}(M)=\bigoplus_{n} \sigma^{*}(M)_{n}$ is the grading of the corresponding twisted module then $\operatorname{sdim} \sigma^{*}(M)_{n}=0$ unless $n-\frac{c}{24}=0$. 


\subsection{Free Fields}

We now describe a free field realization of the small $N=4$ superconformal algebra at $c=6$. Consider the tensor product of the vertex operator superalgebra of four free fermions with the rank four Heisenberg vertex operator algebra. An action of the compact Lie group $S U(2)$ is given by organizing both the fermions and bosons in the standard and conjugate representations of $S U(2)$. By Lemma 3.4 of [17] the sub vertex operator superalgebra of fixed points for this $S U(2)$ action contains the small $N=4$ superconformal algebra at $c=6$. So by using bosonization we obtain a free field realization of the small $N=4$ superconformal algebra at $c=6$ in terms of twelve free fermions. The precise expression for the latter can be found in $\S 2$ of [53. In terms of lattice vertex operator superalgebras this free field realization may be described as follows.

The vertex operator superalgebra of four free fermions may be identified with the lattice vertex operator superalgebra associated to $\mathbb{Z}^{2}$. We have $\mathbb{Z}^{2}=D_{2} \cup\left(D_{2}+[2]\right)$ in the notation of 2.1 , and the exceptional isomorphism $D_{2} \cong A_{1} \oplus A_{1}$. So $V_{D_{2}} \cong$ $V_{A_{1}} \otimes V_{A_{1}}$. Four free fermions are thus the simple current extension of $V_{A_{1}} \otimes V_{A_{1}}$ by the unique simple current with dimension $\frac{1}{2}$. The free field realization in terms of twelve free fermions can be inspected to be a sub vertex operator superalgebra of

$$
V_{D_{2}}^{\otimes 3} \oplus V_{D_{2}+[2]}^{\otimes 3},
$$

and we have just seen that this vertex operator superalgebra is isomorphic to

$$
V_{A_{1}}^{\otimes 6} \oplus V_{A_{1}+(1)}^{\otimes 6}
$$

where $A_{1}+(1)$ denotes the unique non-trivial coset of $A_{1}$ in its dual. For completeness we describe a precise realization. Consider odd fields $b_{1}, \ldots, b_{6}$ and $c_{1}, \ldots, c_{6}$ with operator products

$$
b_{i}(z) c_{j}(w) \sim \frac{\delta_{i, j}}{(z-w)}, \quad b_{i}(z) b_{j}(w) \sim c_{i}(z) c_{j}(w) \sim 0
$$

generating a copy $F(12) \cong V_{\mathbb{Z}^{6}}$ of the vertex operator superalgebra of 12 free fermions. From the above we have $\left(V_{D_{2}} \oplus V_{D_{2}+[2]}\right)^{\otimes 3} \cong V_{\mathbb{Z}^{6}}$. Then $b_{1}, b_{2}, c_{1}, c_{2}$ span the first copy of $V_{D_{2}} \oplus V_{D_{2}+[2]}$, the $b_{3}, b_{4}, c_{3}, c_{4}$ span the second copy, and $b_{5}, b_{6}, c_{5}, c_{6}$ span the last one. The three fields

$$
h=: b_{1} c_{1}:+: b_{2} c_{2}:, \quad e=: b_{1} b_{2}:, \quad f=: c_{1} c_{2}:
$$

are all in the even sub vertex operator algebra $V_{D_{2}}$ of the first copy of $V_{D_{2}} \oplus V_{D_{2}+[2]}$. These three fields strongly generate a vertex operator algebra isomorphic to $L_{1}\left(\mathfrak{s l}_{2}\right)$. 


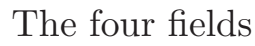

$$
G^{+, 1}=: b_{1} b_{3} b_{5}:, \quad G^{-, 1}=: c_{2} b_{3} b_{5}:, \quad G^{-, 2}=: c_{1} c_{3} c_{5}:, \quad G^{+, 2}=: b_{2} c_{3} c_{5}:,
$$

are all fields in $V_{D_{2}+[2]}^{\otimes 3}$. These seven fields together with the Virasoro field strongly generate a vertex operator superalgebra isomorphic to the small $N=4$ superconfor-

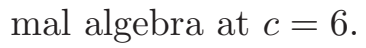

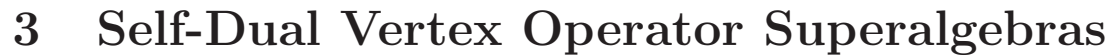

In this section we prove our first main result, which is a classification of self-dual $C_{2}$-cofinite vertex operator superalgebras of CFT type with central charge less than or equal to 12 .

Theorem 3.1. If $W$ is a self-dual $C_{2}$-cofinite vertex operator superalgebr of CFT type with central charge $c \leq 12$ then either $W \cong F(n)$ for some $0 \leq n \leq 24$, or $W \cong V_{E_{8}} \otimes F(n)$ for $0 \leq n \leq 8$, or $W \cong V_{D_{12}^{+}}$.

Proof. We first prove the claimed result for the special case that $c=12$. For this we require to show that a self-dual $C_{2}$-cofinite vertex operator superalgebra of CFT type with $c=12$ is isomorphic to one of $V_{D_{12}^{+}}, V_{E_{8}} \otimes F(8)$ or $F(24)$.

So let $W$ be a self-dual $C_{2}$-cofinite vertex operator superalgebra of CFT type

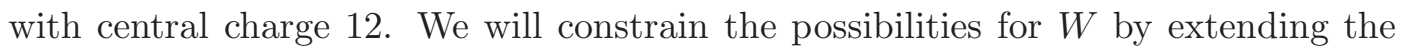
methods used in $\S 5.1$ of [30]. There, $V_{D_{12}^{+}}$is characterized as the unique such vertex

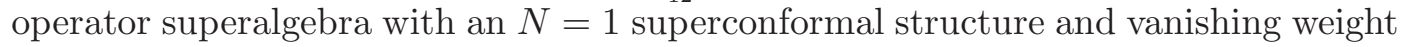
$\frac{1}{2}$ subspace. We will also employ the arguments of $\S 4.2$ of [33], in which the hypothesis

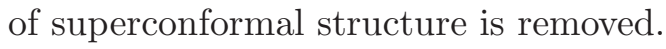

We begin by applying Theorem 2.3 to $W$. In this situation $I_{0}$ and $I_{1}$ are single-

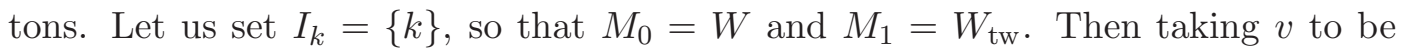
the vacuum in (2.6) we obtain that $Z_{\mathrm{NS}-\mathrm{NS}}^{+}(\tau):=\operatorname{tr}_{W} q^{L_{0}-\frac{1}{2}}$ is a weakly holomorphic modular form with character $\rho_{00}$ of weight 0 for $\Gamma_{\theta}:=\left\langle S, T^{2}\right\rangle$, where $S=\left(\begin{array}{cc}0 & -1 \\ 1 & 0\end{array}\right)$ and $T=\left(\begin{array}{ll}1 & 1 \\ 0 & 1\end{array}\right)$. Further, $\rho_{00}$ is, a priori, trivial on $T^{2}$, and \pm 1 on $S$. This fact is the basis of the proof of Proposition 5.7 in [30], which shows that if $d:=\operatorname{dim} W_{\frac{1}{2}}$ then

$$
\begin{aligned}
Z_{\mathrm{NS}-\mathrm{NS}}^{+}(\tau) & =\frac{\eta(\tau)^{24}}{\eta(2 \tau)^{24}}+24+d \\
& =q^{-\frac{1}{2}}+d+276 q^{\frac{1}{2}}+O(q)
\end{aligned}
$$

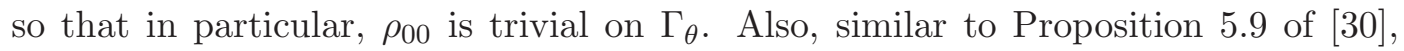
we find from taking $\gamma=T S$ in (2.6) that $\left(W_{\mathrm{tw}}\right)_{n}$ vanishes unless $n \geq \frac{1}{2}$. For another application let $u, u^{\prime} \in W_{1}$. Then taking $v=u_{[-1]} u^{\prime}$ in (2.6), setting $X(\tau):=$ $\operatorname{tr}_{W} O\left(u_{[-1]} u^{\prime}\right) q^{L_{0}-\frac{1}{2}}$ and using the triviality of $\rho_{00}$ on $\Gamma_{\theta}$ we find that $X(\tau)$ is a 
weakly holomorphic modular form of weight 2 for $\Gamma_{\theta}$ satisfying $X(\tau)=C q^{-\frac{1}{2}}+O(1)$ as $\tau \rightarrow i \infty$ for some $C \in \mathbb{C}$. Note that $\Gamma_{\theta}$ has two cusps, represented by $i \infty$ and 1. From (2.6) and the fact that $W_{\text {tw }}$ has $L_{0}$-grading bounded below by $\frac{1}{2}$ we see that $X(\tau)=O(1)$ as $\tau \rightarrow 1$. Since the space of modular forms of weight 2 for $\Gamma_{\theta}$ is one-dimensional, spanned by $\theta_{D_{4}}\left(\frac{\tau+1}{2}\right)=1-24 q^{\frac{1}{2}}+O(q)$ (cf. Theorem 7.1.6 in [73]), it follows that

$$
\begin{aligned}
X(\tau) & =-2 C q \frac{\mathrm{d}}{\mathrm{d} q} Z_{\mathrm{NS}-\mathrm{NS}}^{+}(\tau)+D \theta_{D_{4}}\left(\frac{\tau+1}{2}\right) \\
& =C q^{-\frac{1}{2}}+D+(-276 C-24 D) q^{\frac{1}{2}}+O(q)
\end{aligned}
$$

for some $C, D \in \mathbb{C}$ (with $C$ as above), which we can expect will depend on $u$ and $u^{\prime}$.

We now endeavour to connect $C$ and $D$ in (3.2) to the Lie algebra structure on $W_{1}$. For this note that the first paragraph of the proof of Theorem 4.5 in 33 ] applies to $W$, showing that $W$ admits a unique (up to scale) non-degenerate invariant bilinear form, which we henceforth denote $\langle\cdot, \cdot\rangle$. We also have that $W_{1}$ is contained in the kernel of $L_{1}$, so by Theorem 1.1 of [26] the Lie algebra structure on $W_{1}$ is reductive. Applying the argument of the proof of Theorem 5.12 in [30] to $W$ - this uses the identity (3.1) - we see that the Lie rank of $W_{1}$ is bounded above by 12 . We can identify a simple component of $W_{1}$ just by considering $d=\operatorname{dim} W_{\frac{1}{2}}$. To do this note that the invariant bilinear form $\langle\cdot, \cdot\rangle$ on $W$ is non-degenerate when restricted to $W_{\frac{1}{2}}$. If we let $U$ denote the sub vertex operator superalgebra of $W$ generated by $W_{\frac{1}{2}}$ then, arguing as in [55] (cf. also $\S 3$ of [77]), we see that $U$ is isomorphic to the Clifford algebra vertex operator superalgebra defined by the orthogonal space structure on $W_{\frac{1}{2}}$, and if $V$ is the commutant of $U$ in $W$ then $W$ is naturally isomorphic to $U \otimes V$. So $W_{1}$ contains $U_{1}$, which is the Lie algebra naturally associated to the orthogonal structure on $W_{\frac{1}{2}}$. Since $W_{1}$ has Lie rank bounded above by 12 we must have $d \leq 24$. Indeed, the case that $d=24$ is realized by $W=U=F(24)$.

Table 1: Dual Coxeter numbers of simple complex Lie algebras

\begin{tabular}{|c|c|}
\hline $\mathfrak{g}$ & $h^{\vee}$ \\
\hline$A_{n}$ & $n+1$ \\
$B_{n}$ & $2 n-1$ \\
$C_{n}$ & $n+1$ \\
$D_{n}$ & $2 n-2$ \\
$E_{6}$ & 12 \\
$E_{7}$ & 18 \\
$E_{8}$ & 30 \\
$F_{4}$ & 9 \\
$G_{2}$ & 4 \\
\hline
\end{tabular}

So suppose henceforth that $d<24$. Then $\operatorname{dim} U_{1}<\operatorname{dim} W_{1}=276$, and $V_{1} \neq\{0\}$. 
To further constrain $W_{1}$ we consider (3.2) with $u, u^{\prime} \in V_{1}$. Note that since $V_{\frac{1}{2}}=\{0\}$ by construction we have $\operatorname{tr}_{W} o(u) o\left(u^{\prime}\right) q^{L_{0}-\frac{1}{2}}=\kappa\left(u, u^{\prime}\right) q^{\frac{1}{2}}+O(q)$ where $\kappa$ is the Killing form on $W_{1}$. We have $u_{[1]} u^{\prime}=\left\langle u, u^{\prime}\right\rangle \mathbf{v}$ according to Lemma 5.1 of [30], so by an application of Sublemma 6.9 of [28] we find that

$$
\begin{aligned}
X(\tau) & =\operatorname{tr}_{W} o(u) o\left(u^{\prime}\right) q^{L_{0}-\frac{1}{2}}-\frac{1}{12}\left\langle u, u^{\prime}\right\rangle E_{2}(\tau) Z_{\mathrm{NS}-\mathrm{NS}}^{+}(\tau) \\
& =-\frac{1}{12}\left\langle u, u^{\prime}\right\rangle q^{-\frac{1}{2}}-\frac{1}{12}\left\langle u, u^{\prime}\right\rangle d+\left(\kappa\left(u, u^{\prime}\right)-21\left\langle u, u^{\prime}\right\rangle\right) q^{\frac{1}{2}}+O(q)
\end{aligned}
$$

where $E_{2}(\tau)=1-24 \sum_{n>0} \frac{n q^{n}}{1-q^{n}}$ is the quasi-modular Eisenstein series of weight 2 , and $Z_{\mathrm{NS}-\mathrm{NS}}^{+}(\tau)$ is as in (3.1). Comparing (3.2) with (3.3) we find that $C=-\frac{1}{12}\left\langle u, u^{\prime}\right\rangle$, $D=-\frac{1}{12}\left\langle u, u^{\prime}\right\rangle d$, and

$$
\kappa\left(u, u^{\prime}\right)=(44+2 d)\left\langle u, u^{\prime}\right\rangle
$$

In particular, the Killing form is non-degenerate on $V_{1}$, so the Lie algebra structure on $V_{1}$ is semisimple. So let $\mathfrak{g}$ be a simple component of $V_{1}$. From the main theorem of [27] we know that the vertex operators attached to $V_{1}$ represent the affine Lie algebra associated to $\mathfrak{g}$ with integral level; call it $k$. Then for $\alpha$ a long root of $\mathfrak{g}$ we have $\kappa(\alpha, \alpha)=4 h$ where $h$ is the dual Coxeter number of $\mathfrak{g}$, and $\langle\alpha, \alpha\rangle=2 k$. So (3.4) with $u=u^{\prime}=\alpha$ yields $h=(22+d) k$. We are reduced to finding the pairs $(d, \mathfrak{g})$ where $d$ is an integer $0 \leq d<24$, and $\mathfrak{g}$ is a simple Lie algebra with Lie rank bounded above by $12-\frac{1}{2} d$ such that the dual Coxeter number $h^{\vee}$ of $\mathfrak{g}$ is an integer multiple of $22+d$. Inspecting Table 1 we see that either $d=0$ and $\mathfrak{g}$ is of type $D_{12}$, or $d=8$ and $\mathfrak{g}$ is of type $E_{8}$. The first of these is realized by $W=V_{D_{12}^{+}}$. The second is realized by $W=V_{E_{8}} \otimes F(8)$. Thus we have dealt with the special case that $c=12$.

To complete the proof let $W$ be a self-dual $C_{2}$-cofinite vertex operator superalgebra of CFT type with $c<12$. By applying Theorem 11.3 of [23] to $W^{\text {even }}$ we may conclude that $c$ is a rational number. Then the argument of Satz 2.2.2 of [62] applies to $W$, and shows that $c \in \frac{1}{2} \mathbb{Z}$. So $n=24-2 c$ is a positive integer and $W^{\prime}:=W \otimes F(n)$ is a self-dual $C_{2}$-cofinite vertex operator superalgebra of CFT type with central charge $c^{\prime}=12$. Since $n$ is positive $W^{\prime}$ is one of $V_{E_{8}} \otimes F(8)$ or $F(24)$, by what we have already proved about self-dual vertex operator superalgebras with central charge 12. The desired conclusion follows. 


\section{Superconformal Field Theory}

\subsection{Potential Bulk Conformal Field Theory}

The basic structure underlying a bulk conformal field theory is a module $\mathcal{H}$ for a tensor product $V^{\prime} \otimes V^{\prime \prime}$ of vertex operator algebras $V^{\prime}$ and $V^{\prime \prime}$. It is required that

$$
\mathcal{H}=\bigoplus_{i} N_{i}^{\prime} \otimes N_{i}^{\prime \prime}
$$

where the $N_{i}^{\prime}$ and $N_{i}^{\prime \prime}$ are irreducible modules for $V^{\prime}$ and $V^{\prime \prime}$, respectively. Also, $V^{\prime} \otimes V^{\prime \prime}$ should appear exactly once as a summand of $\mathcal{H}$. A standard but very special case is that $V^{\prime}=V^{\prime \prime}$ is rational and $C_{2}$-cofinite, and the $N_{i}^{\prime}=N_{i}^{\prime \prime}$ are all the irreducible $V^{\prime}$-modules. We call this the diagonal conformal field theory for $V=V^{\prime}=V^{\prime \prime}$.

Various further properties are required of $\mathcal{H}$, including closure under fusion and modular invariance. Define

$$
\begin{aligned}
\widehat{\operatorname{ch}}[\mathcal{H}]\left(\tau^{\prime}, \tau^{\prime \prime}\right): & =\operatorname{tr}_{\mathcal{H}}\left(q^{\prime L_{0}^{\prime}-\frac{c^{\prime}}{24}} q^{\prime \prime L_{0}^{\prime \prime}-\frac{c^{\prime \prime}}{24}}\right) \\
& =\sum_{i} \operatorname{ch}\left[N_{i}^{\prime}\right]\left(\tau^{\prime}\right) \operatorname{ch}\left[N_{i}^{\prime \prime}\right]\left(\tau^{\prime \prime}\right)
\end{aligned}
$$

(cf. (2.10)). Modular invariance is the requirement that the partition function

$$
\begin{aligned}
Z_{\mathcal{H}}(\tau): & =\widehat{\operatorname{ch}}[\mathcal{H}](\tau,-\bar{\tau}) \\
& =\sum_{i} \operatorname{ch}\left[N_{i}^{\prime}\right](\tau) \operatorname{ch}\left[N_{i}^{\prime \prime}\right](-\bar{\tau})
\end{aligned}
$$

be invariant for the natural action of $S L_{2}(\mathbb{Z})$. Closure under fusion is the requirement that operator products close on $\mathcal{H}$. So superficially at least, fusion and modularity force a bulk conformal field theory to resemble a self-dual vertex operator algebra. This is the motivation for the Main Question we formulated in $\$ 1$.

We will answer the Main Question positively in what follows, using a certain convenient substitute for the notion of bulk conformal field theory. Also, we will find that there are more examples if we allow the vertex operator algebra in the question to be a vertex operator superalgebra.

To motivate our approach note that modular invariance is a strong constraint that is used in practice to classify possible examples of conformal field theories. There are additional properties of correlation functions that are harder to verify (cf. [82, 87]), but from a representation category point of view these correlation requirements are satisfied by symmetric special Frobenius algebra objects in the modular tensor category of a suitably chosen vertex operator algebra according to 477. In this work lattice vertex operator algebras underly all examples, so all simple 
objects are simple currents, and if a symmetric special Frobenius algebra object $\mathcal{A}$ is a direct sum of inequivalent simple currents then there is a rather explicit prescription for the decomposition (4.1); namely (5.85) of [47]. For example, if we assume $V^{\prime} \cong V^{\prime \prime}$ and choose $\mathcal{A}=V^{\prime}$ then the partition function of the bulk is the charge conjugation invariant. In the cases we consider every irreducible $V^{\prime}$-module will be invariant under charge conjugation, so the charge conjugation invariant will coincide with the ordinary diagonal modular invariant. Motivated by this we introduce the following.

Definition 4.1. A potential bulk conformal field theory is a $V^{\prime} \otimes V^{\prime \prime}$-module $\mathcal{H}$ as in (4.1) such that the partition function (4.3) is modular invariant.

Now to formulate an answer to the Main Question, suppose that $W$ is a self-dual $C_{2}$-cofinite vertex operator superalgebra such that

$$
W \cong \bigoplus_{i} N_{i}^{\prime} \otimes N_{i}^{\prime \prime}
$$

as a $V^{\prime} \otimes V^{\prime \prime}$-module, for $V^{\prime}$ and $V^{\prime \prime}$ a commuting pair of rational $C_{2}$-cofinite sub vertex operator algebras, where the $N_{i}^{\prime}$ and $N_{i}^{\prime \prime}$ are irreducible modules for $V^{\prime}$ and $V^{\prime \prime}$, respectively. Define

$$
Z_{W}(\tau):=\widehat{\operatorname{ch}}^{+}[W](\tau,-\bar{\tau})
$$

where $\widehat{c h}^{ \pm}[\cdot]$ is as in (2.10).

Proposition 4.2. With $W$ as in 4.4), if the $S$-matrix of $V^{\prime \prime}$ is real and the eigenvalues of the action of $L_{0}^{\prime}-L_{0}^{\prime \prime}$ on $W$ belong to $\mathbb{Z}+\frac{1}{24}\left(c^{\prime}-c^{\prime \prime}\right)$ then $Z_{W}$ is modular invariant.

Proof. Since $W$ is self-dual Theorem 2.3 implies that $\mathrm{ch}^{+}[W](\mathrm{cf} .(2.8)$ ) is invariant under $S$. It follows from Theorem 2.4 then that $\widehat{c h}^{+}[W]\left(\tau^{\prime}, \tau^{\prime \prime}\right)$ is $S$-invariant as well. In particular we have

$$
\begin{aligned}
\widehat{\operatorname{ch}}^{+}[W]\left(-\frac{1}{\tau^{\prime}},-\frac{1}{\tau^{\prime \prime}}\right) & =\sum_{i, j, \ell} S_{i j}^{\prime} \operatorname{ch}^{+}\left[N_{j}^{\prime}\right]\left(\tau^{\prime}\right) S_{i \ell}^{\prime \prime} \operatorname{ch}^{+}\left[N_{\ell}^{\prime \prime}\right]\left(\tau^{\prime \prime}\right) \\
& =\sum_{i} \operatorname{ch}^{+}\left[N_{i}^{\prime}\right]\left(\tau^{\prime}\right) \operatorname{ch}^{+}\left[N_{i}^{\prime \prime}\right]\left(\tau^{\prime \prime}\right)
\end{aligned}
$$

for suitable matrices $S^{\prime}$ and $S^{\prime \prime}$. Consider the action of $S$ on $Z_{W}(\tau)$. Using (4.6) and 
the hypothesis that the entries of $S^{\prime \prime}$ are real we compute

$$
\begin{aligned}
Z_{W}\left(-\frac{1}{\tau}\right) & =\sum_{i} \operatorname{ch}^{+}\left[N_{i}^{\prime}\right]\left(-\frac{1}{\tau}\right) \overline{\operatorname{ch}^{+}\left[N_{i}^{\prime \prime}\right]\left(-\frac{1}{\tau}\right)} \\
& =\sum_{i, j, \ell} S_{i, j}^{\prime} \operatorname{ch}^{+}\left[N_{j}^{\prime}\right](\tau) \overline{S_{i, \ell}^{\prime \prime} \operatorname{ch}^{+}\left[N_{\ell}^{\prime \prime}\right](\tau)} \\
& =\sum_{i, j, \ell} S_{i, j}^{\prime} \operatorname{ch}^{+}\left[N_{j}^{\prime}\right](\tau) S_{i, \ell}^{\prime \prime} \operatorname{ch}^{+}\left[N_{\ell}^{\prime \prime}\right](-\bar{\tau}) \\
& =\sum_{i} \operatorname{ch}^{+}\left[N_{i}^{\prime}\right](\tau) \operatorname{ch}^{+}\left[N_{i}^{\prime \prime}\right](-\bar{\tau}) \\
& =Z_{W}(\tau) .
\end{aligned}
$$

So $Z_{W}$ is $S$-invariant. Invariance under $T$ follows from

$$
\begin{aligned}
Z_{W}(\tau+1) & =\operatorname{tr}_{W}\left(e^{2 \pi i(\tau+1)\left(L_{0}^{\prime}-\frac{c^{\prime}}{24}\right)} e^{-2 \pi i(\bar{\tau}+1)\left(L_{0}^{\prime \prime}-\frac{c^{\prime \prime}}{24}\right)}\right) \\
& =e^{\frac{2 \pi i}{24}\left(c^{\prime \prime}-c^{\prime}\right)} \operatorname{tr}_{W}\left(e^{2 \pi i\left(L_{0}^{\prime}-L_{0}^{\prime \prime}\right)} q^{L_{0}^{\prime}-\frac{c^{\prime}}{24}} \bar{q}^{L_{0}^{\prime \prime}-\frac{c^{\prime \prime}}{24}}\right)
\end{aligned}
$$

and our hypothessis on the eigenvalues of $L_{0}^{\prime}$ and $L_{0}^{\prime \prime}$. This completes the proof.

Proposition 4.2 gives a path to answering the Main Question positively, in the sense that if its hypotheses are satisfied then it identifies a self-dual vertex operator superalgebra $W$ with a potential bulk conformal field theory $\mathcal{H}$ as a module for the underlying vertex operator algebra $V^{\prime} \otimes V^{\prime \prime}$. The two conditions of Proposition 4.2 are strong, but are satisfied in interesting cases as we will see in 95 .

\subsection{Potential Bulk Superconformal Field Theory}

We are also interested in relating self-dual vertex operator superalgebras to superconformal field theories in this work. To define a supersymmetric counterpart to the notion of potential bulk conformal field theory we consider a $V^{\prime} \otimes V^{\prime \prime}$-module

$$
\mathcal{H}=\bigoplus_{i} N_{i}^{\prime} \otimes N_{i}^{\prime \prime}
$$

as in (4.1), but allow $V^{\prime}$ and $V^{\prime \prime}$ to be vertex operator superalgebras, and allow the $N_{i}^{\prime}$ in (4.1) to be irreducible untwisted or canonically twisted modules for $V^{\prime}$, and similarly for the $N_{i}^{\prime \prime}$. (Strictly speaking, $\mathcal{H}$ is a module for the even sub vertex operator algebra of $V^{\prime} \otimes V^{\prime \prime}$.) Following tradition we use subscripts NS and R to indicate restrictions to untwisted and canonically twisted modules for $V^{\prime}$ and $V^{\prime \prime}$. 
So,

$$
\begin{aligned}
\mathcal{H}_{\mathrm{NS}-\mathrm{NS}}:= & \bigoplus_{\substack{i \\
N_{i}^{\prime} \text { untwisted } \\
N_{i}^{\prime \prime} \text { untwisted }}} N_{i}^{\prime} \otimes N_{i}^{\prime \prime}, \\
\mathcal{H}_{\mathrm{NS}-\mathrm{R}}:= & \bigoplus_{\substack{i \\
N_{i}^{\prime} \text { untwisted } \\
N_{i}^{\prime \prime} \text { twisted }}} N_{i}^{\prime} \otimes N_{i}^{\prime \prime},
\end{aligned}
$$

and so on. We call $\mathcal{H}_{\text {NS-NS }}$ the NS-NS sector, \&c. We also assume that $\mathcal{H}$ is equipped with a compatible superspace structure $\mathcal{H}=\mathcal{H}^{\text {even }} \oplus \mathcal{H}^{\text {odd }}$, so that $\mathcal{H}$ is graded by $(\mathbb{Z} / 2 \mathbb{Z})^{3}$

$$
\mathcal{H}=\mathcal{H}_{\mathrm{NS}-\mathrm{NS}}^{\text {even }} \oplus \mathcal{H}_{\mathrm{NS}-\mathrm{NS}}^{\text {odd }} \oplus \mathcal{H}_{\mathrm{NS}-\mathrm{R}}^{\text {even }} \oplus \cdots \oplus \mathcal{H}_{\mathrm{R}-\mathrm{R}}^{\text {even }} \oplus \mathcal{H}_{\mathrm{R}-\mathrm{R}}^{\text {odd }}
$$

We may regard a bulk conformal field theory as a bulk superconformal field theory in which only the even part of the NS-NS sector is non-zero. From this point of view it is natural to expect examples in which the NS-NS sector of a superconformal field theory is identified with a self-dual vertex operator superalgebra, and the R-R sector is identified with its canonically twisted module. As such, the prescription (4.3) does not usually define a modular invariant function when the superspace structure is non-trivial. Rather, Theorem 2.3 indicates that we should consider the vectorvalued function

$$
Z_{\mathcal{H}}(\tau):=\left(\begin{array}{c}
Z_{\mathrm{NS}-\mathrm{NS}}^{+}(\tau) \\
Z_{\mathrm{NS}-\mathrm{NS}}^{-}(\tau) \\
Z_{\mathrm{R}-\mathrm{R}}^{+}(\tau) \\
Z_{\mathrm{R}-\mathrm{R}}^{-}(\tau)
\end{array}\right)
$$

where $Z_{\mathrm{X}-\mathrm{Y}}^{ \pm}$is defined by setting $Z_{\mathrm{X}-\mathrm{Y}}^{ \pm}(\tau):=\widehat{c h}^{ \pm}\left[\mathcal{H}_{\mathrm{X}-\mathrm{Y}}\right](\tau,-\bar{\tau})(\mathrm{cf}$. (2.10) $)$ for $\mathrm{X}, \mathrm{Y} \in$ $\{\mathrm{NS}, \mathrm{R}\}$. Then modularity for $Z_{\mathcal{H}}$ is the requirement that

$$
\begin{aligned}
& \mathbf{S} \cdot Z_{\mathcal{H}}\left(-\frac{1}{\tau}\right)=Z_{\mathcal{H}}(\tau), \\
& \mathbf{T} \cdot Z_{\mathcal{H}}(\tau+1)=Z_{\mathcal{H}}(\tau),
\end{aligned}
$$

where

$$
\mathbf{S}:=\left(\begin{array}{cccc}
1 & 0 & 0 & 0 \\
0 & 0 & 1 & 0 \\
0 & 1 & 0 & 0 \\
0 & 0 & 0 & 1
\end{array}\right), \quad \mathbf{T}:=\left(\begin{array}{llll}
0 & 1 & 0 & 0 \\
1 & 0 & 0 & 0 \\
0 & 0 & 1 & 0 \\
0 & 0 & 0 & 1
\end{array}\right)
$$


Motivated by this we formulate the following super-analogue of Definition 4.1.

Definition 4.3. A potential bulk superconformal field theory is a $V^{\prime} \otimes V^{\prime \prime}$-module $\mathcal{H}$ as in (4.9) whose partition function (4.13) satisfies (4.14).

Remark 4.4. For bulk superconformal field theories we expect that correlation function requirements are encoded by a suitably formulated notion of symmetric special Frobenius superalgebra object. It would be interesting to generalize [47 to the super setting, and determine whether the examples we describe in this work are compatible or not. Superalgebra objects are introduced in the context of vertex algebra tensor categories in [18, 19].

To formulate a supersymmetric counterpart to Proposition 4.2 consider a self-dual $C_{2}$-cofinite vertex operator superalgebra $W$ such that

$$
W \cong \bigoplus_{i} N_{i}^{\prime} \otimes N_{i}^{\prime \prime}
$$

as a $V^{\prime} \otimes V^{\prime \prime}$-module, for $V^{\prime}$ and $V^{\prime \prime}$ a commuting pair of rational $C_{2}$-cofinite sub vertex operator superalgebras, where the $N_{i}^{\prime}$ and $N_{i}^{\prime \prime}$ are irreducible (untwisted) modules for $V^{\prime}$ and $V^{\prime \prime}$, respectively. Write $W_{\text {tw }}$ for the unique irreducible canonically twisted $W$-module (cf. 92.1) and chose a superspace structure $W_{\mathrm{tw}}=W_{\mathrm{tw}}^{\text {even }} \oplus W_{\mathrm{tw}}^{\mathrm{odd}}$ that is compatible with the superspace structure on $W$. We then define $Z_{W}(\tau)$ in analogy with (4.13), so that

$$
Z_{W}(\tau):=\left(\begin{array}{c}
Z^{+}(\tau) \\
Z^{-}(\tau) \\
Z_{\mathrm{tw}}^{+}(\tau) \\
Z_{\mathrm{tw}}^{-}(\tau)
\end{array}\right)
$$

where $Z^{ \pm}(\tau):=\widehat{\operatorname{ch}}^{ \pm}[W](\tau,-\bar{\tau})$ and $Z_{\mathrm{tw}}^{ \pm}(\tau):=\widehat{\mathrm{ch}}^{ \pm}\left[W_{\mathrm{tw}}^{\text {even }}\right](\tau,-\bar{\tau})$. The proof of the next result follows in a directly similar way to that of Proposition 4.2 .

Proposition 4.5. With $W$ as in (4.16), if the $S$-matrix of $V^{\prime \prime}$ is real, the eigenvalues of $L_{0}^{\prime}-L_{0}^{\prime \prime}$ on $W^{\text {even }}$ lie in $\mathbb{Z}+\frac{1}{24}\left(c^{\prime}-c^{\prime \prime}\right)$, and the eigenvalues of $L_{0}^{\prime}-L_{0}^{\prime \prime}$ on $W^{\text {odd }}$ lie in $\mathbb{Z}+\frac{1}{2}+\frac{1}{24}\left(c^{\prime}-c^{\prime \prime}\right)$ then $Z_{W}$ satisfies the modularity condition 4.14).

Thus a vertex operator superalgebra $W$ satisfying the hypotheses of Proposition 4.5 also answers the Main Question positively, in the sense that it is identified with the NS-NS sector of a potential bulk superconformal field theory as a module for the underlying vertex operator superalgebra $V^{\prime} \otimes V^{\prime \prime}$, and similarly for $W_{\mathrm{tw}}$ and the R-R sector. Note that reality of the modular $S$-matrix holds for $L_{k}\left(\mathfrak{s l}_{2}\right)$ when $k$ is positive and integral, and holds also for minimal models of the Virasoro algebra. Inspecting Weil's description of the modular group action on theta functions for cosets of an 
even lattice $L$ in its dual $L^{*}$, we see that if the inner products on $L^{*}$ are contained in $\frac{1}{2} \mathbb{Z}$ then the $S$-matrix for the lattice vertex operator algebra $V_{L}$ is also real. This holds in particular for $L=D_{4 n}$, which will play a prominent role in what follows.

\subsection{Superconformal structure}

Superconformal field theories are usually assumed to come equipped with supersymmetry. With $\mathcal{H}$ as in (4.9) we define an $N=(2,2)$ superconformal structure to be an identification of sub vertex operator superalgebras of $V^{\prime}$ and $V^{\prime \prime}$ with vacuum modules for the $N=2$ superconformal algebra. In this situation it is natural to consider

$$
Z_{\mathcal{H}}(u, \tau):=\left(\begin{array}{c}
Z_{\mathrm{NS}-\mathrm{NS}}^{+}(u, \tau) \\
Z_{\mathrm{NS}-\mathrm{NS}}^{-}(u, \tau) \\
Z_{\mathrm{R}-\mathrm{R}}^{+}(u, \tau) \\
Z_{\mathrm{R}-\mathrm{R}}^{-}(u, \tau)
\end{array}\right)
$$

where $Z_{\mathrm{X}-\mathrm{Y}}^{ \pm}(u, \tau):=\widehat{\operatorname{ch}}^{ \pm}\left[\mathcal{H}_{\mathrm{X}-\mathrm{Y}}\right](u, \tau,-\bar{u},-\bar{\tau})$ and

$$
\widehat{c h}^{ \pm}[M]\left(u^{\prime}, \tau^{\prime}, u^{\prime \prime}, \tau^{\prime \prime}\right):=\operatorname{tr}_{M}\left(( \pm 1)^{F} z^{\prime J_{0}^{\prime}} q^{L_{0}^{\prime}-\frac{c^{\prime}}{24}} z^{\prime \prime} J_{0}^{\prime \prime} q^{\prime \prime L_{0}^{\prime \prime}-\frac{c^{\prime \prime}}{24}}\right) .
$$

Here $z^{\prime}=e^{2 \pi i u^{\prime}}$ and $q^{\prime}=e^{2 \pi i \tau^{\prime}}$, \&c. We also have the elliptic genus of $\mathcal{H}$, defined by setting

$$
E_{\mathcal{H}}(u, \tau):=\widehat{\operatorname{ch}}^{-}\left[\mathcal{H}_{\mathrm{R}-\mathrm{R}}\right](u, \tau, 0,-\bar{\tau}) .
$$

Now the modularity conditions on $\mathcal{H}$ are richer. For simplicity we describe them in the special case that $c^{\prime}=c^{\prime \prime}$. Then the natural counterpart to (4.14) is

$$
\begin{aligned}
e^{-\pi i \frac{c}{6}\left(\frac{u^{2}}{\tau}-\frac{\bar{u}^{2}}{\bar{\tau}}\right)} \mathbf{S} \cdot Z_{\mathcal{H}}\left(\frac{u}{\tau},-\frac{1}{\tau}\right) & =Z_{\mathcal{H}}(u, \tau), \\
\mathbf{T} \cdot Z_{\mathcal{H}}(u, \tau+1) & =Z_{\mathcal{H}}(u, \tau),
\end{aligned}
$$

where $c=c^{\prime}=c^{\prime \prime}$, and $\mathbf{S}$ and $\mathbf{T}$ are as in (4.15). Call this modularity for $Z_{\mathcal{H}}$. If $\mathcal{H}$ is a superconformal field theory underlying a sigma model with Calabi-Yau target space $X$ then $E_{\mathcal{H}}$ is also modular, in the sense that we have

$$
\begin{aligned}
e^{-\pi i d \frac{u^{2}}{\tau}} E_{\mathcal{H}}\left(\frac{u}{\tau},-\frac{1}{\tau}\right) & =E_{\mathcal{H}}(u, \tau), \\
E_{\mathcal{H}}(u, \tau+1) & =E_{\mathcal{H}}(u, \tau),
\end{aligned}
$$

where $d=\operatorname{dim}_{\mathbb{C}} X$, and $\tau \mapsto E_{\mathcal{H}}(u, \tau)$ remains bounded as $\tau \rightarrow i \infty$, for any fixed $u \in \mathbb{C}$. That is, $E_{\mathcal{H}}$ is a weak Jacobi form of weight 0 and index $\frac{1}{2} \operatorname{dim}_{\mathbb{C}} X$. In 
this situation $E_{\mathcal{H}}(0, \tau)$ is the Euler characteristic of $X$. (In particular, $E_{\mathcal{H}}(0, \tau)$ is a constant function of $\tau$.) Let us say that $\mathcal{H}$ is elliptic if $E_{\mathcal{H}}$ satisfies (4.22). Say that $\mathcal{H}$ satisfies spectral flow symmetry if

$$
Z_{\mathrm{R}-\mathrm{R}}^{ \pm}\left(u^{\prime}, \tau^{\prime}, u^{\prime \prime}, \tau^{\prime \prime}\right)=\left(z^{\prime} z^{\prime \prime}\right)^{\frac{c}{6}}\left(q^{\prime} q^{\prime \prime}\right)^{\frac{c}{24}} Z_{\mathrm{NS}-\mathrm{NS}}^{ \pm}\left(u^{\prime}+\frac{1}{2} \tau^{\prime}, \tau^{\prime}, u^{\prime \prime}+\frac{1}{2} \tau^{\prime \prime}, \tau^{\prime \prime}\right)
$$

where $c=c^{\prime}=c^{\prime \prime}$.

At this point it is natural to define a potential bulk $N=(2,2)$ superconformal field theory to be a potential bulk superconformal field theory $\mathcal{H}$ (cf. Definition 4.3 ) with $N=(2,2)$ superconformal structure such that (4.21), (4.22) and (4.23) are satisfied. These are strict requirements. Interestingly, we will see in examples that the extra superconformal structure allows us to weaken these requirements in what seems to be a useful way. In anticipation of this we offer the following.

Definition 4.6. Say that $\mathcal{H}$ as in (4.9) is a quasi potential bulk $N=(2,2)$ superconformal field theory if $\mathcal{H}$ is elliptic (4.22), satisfies spectral flow symmetry (4.23), and if $Z_{\mathcal{H}}$ is modular (4.21) for some finite index subgroup of the modular group.

So for the notion of quasi potential bulk $N=(2,2)$ superconformal field theory we relax condition (4.21), which is the invariance of $Z_{\mathcal{H}}$ under the action of $S L_{2}(\mathbb{Z})$ defined by the left hand sides of (4.21), and require invariance only for some subgroup $\Gamma<S L_{2}(\mathbb{Z})$ such that the coset space $\Gamma \backslash S L_{2}(\mathbb{Z})$ is finite.

We may also be interested in the case that both $V^{\prime}$ and $V^{\prime \prime}$ contain the small $N=4$ superconformal algebra at some central charge $c$. In this situation we call $\mathcal{H}$ a potential bulk $N=(4,4)$ superconformal field theory if (4.21), (4.22) and (4.23) hold, and if in addition $c=6 k$ for $k$ a positive integer, and spectral flow for each of $V^{\prime}$ and $V^{\prime \prime}$ is realized by fusion with the order two simple current of $L_{k}\left(\mathfrak{s l}_{2}\right.$ ) (as discussed in 2.3$)$. For the notion of quasi potential bulk $N=(4,4)$ superconformal field theory we relax the requirement of modularity (4.21) of $Z_{\mathcal{H}}$ from the modular group to some finite index subgroup.

In order to present a counterpart to Proposition 4.5 we now consider a self-dual $C_{2}$-cofinite vertex operator superalgebra $W$ with a decomposition as in (4.16) such that $V^{\prime}$ and $V^{\prime \prime}$ contain copies of the vacuum module for the $N=2$ superconformal algebra at some central charge $c=c^{\prime}=c^{\prime \prime}$. We then define $Z_{W}(u, \tau)$ in analogy with (4.18), setting

$$
Z_{W}(u, \tau):=\left(\begin{array}{c}
Z^{+}(u, \tau) \\
Z^{-}(u, \tau) \\
Z_{\mathrm{tw}}^{+}(u, \tau) \\
Z_{\mathrm{tw}}^{-}(u, \tau)
\end{array}\right)
$$

where $Z^{ \pm}(u, \tau):=\widehat{\mathrm{ch}}^{ \pm}[W](u, \tau,-\bar{u},-\bar{\tau})$ and $Z_{\mathrm{tw}}^{ \pm}(u, \tau):=\widehat{\mathrm{ch}}^{ \pm}\left[W_{\mathrm{tw}}^{\mathrm{even}}\right](u, \tau,-\bar{u},-\bar{\tau})$, 
and $\widehat{c h}^{ \pm}[\cdot]$ is as in (4.19). The proof of the next result is similar to the proofs of Propositions 4.2 and 4.5, but note the restriction that $c^{\prime}=c^{\prime \prime}$.

Proposition 4.7. Suppose that $W$ is as in 4.16), and $V^{\prime}$ and $V^{\prime \prime}$ contain the vacuum module for the $N=2$ superconformal algebra at some central charge $c=c^{\prime}=c^{\prime \prime}$. If $L_{0}^{\prime}-L_{0}^{\prime \prime}$ acts on $W^{\text {even }}$ and $W_{\mathrm{tw}}$ with eigenvalues in $\mathbb{Z}$, and on $W^{\text {odd }}$ with eigenvalues in $\mathbb{Z}+\frac{1}{2}$, and if the $S$-matrices of $V^{\prime}$ and $V^{\prime \prime}$ are both real, then $Z_{W}$ satisfies the modularity and spectral flow symmetry conditions, 4.21) and 4.23).

Define the elliptic genus of $W$ by setting

$$
E_{W}(u, \tau):=\widehat{c h}^{-}\left[W_{\mathrm{tw}}\right](u, \tau, 0,-\bar{\tau})
$$

We will presently see examples $W$ for which Proposition 4.7 fails but spectral flow symmetry holds and $E_{W}$ is a weak Jacobi form of weight 0 and some index.

\section{$5 \quad$ Examples}

\subsection{Type D Conformal Field Theory}

Let $n$ be a positive integer. The bulk of the diagonal conformal field theory wth $V^{\prime}=V^{\prime \prime}=V_{D_{2 n}}$ is

$$
\mathcal{H}=\bigoplus_{i=0}^{3} V_{D_{2 n}+[i]} \otimes V_{D_{2 n}+[i]} .
$$

Observe that we may embed $D_{2 n} \oplus D_{2 n}$ in $D_{4 n}$ by taking the first copy of $D_{2 n}$ to be the vectors in $D_{4 n}$ supported on the first $2 n$ components, and letting the second copy be its orthogonal complement. Then for the cosets of $D_{4 n}$ in its dual we have

$$
\begin{aligned}
& D_{4 n}+[0]=\left(D_{2 n}+[0], D_{2 n}+[0]\right) \cup\left(D_{2 n}+[2], D_{2 n}+[2]\right), \\
& D_{4 n}+[1]=\left(D_{2 n}+[1], D_{2 n}+[1]\right) \cup\left(D_{2 n}+[3], D_{2 n}+[3]\right), \\
& D_{4 n}+[2]=\left(D_{2 n}+[0], D_{2 n}+[2]\right) \cup\left(D_{2 n}+[2], D_{2 n}+[0]\right), \\
& D_{4 n}+[3]=\left(D_{2 n}+[1], D_{2 n}+[3]\right) \cup\left(D_{2 n}+[3], D_{2 n}+[1]\right) .
\end{aligned}
$$

From this we immediately obtain the following result.

Proposition 5.1. For $n$ a positive integer, the bulk of the diagonal $V_{D_{2 n}}$ conformal field theory is isomorphic to $V_{D_{4 n}^{+}}$as a $V_{D_{2 n}} \otimes V_{D_{2 n}}$-module.

Note that $D_{4}^{+} \simeq \mathbb{Z}^{4}$ and $D_{8}^{+} \simeq E_{8}$. So Proposition 5.1 furnishes bulk conformal field theory interpretations for the self-dual vertex operator superalgebras $F(8), V_{E_{8}}$ and $V_{D_{12}^{+}}$appearing in Theorem 3.1 . 
An embedding of $A_{1}^{2 n}$ in $D_{2 n}$ is discussed in 2.1. We may use this to formulate a counterpart to Proposition 5.1 for tensor powers of $L_{1}\left(\mathfrak{s l}_{2}\right)$. For example, if $\mathcal{H}$ denotes the bulk of the diagonal conformal field theory of $L_{1}\left(\mathfrak{s l}_{2}\right)^{\otimes 2 n}$ then by virtue of the isomorphism $V_{A_{1}} \cong L_{1}\left(\mathfrak{s l}_{2}\right)$ we have

$$
\begin{aligned}
\mathcal{H} & =\bigoplus_{C \in \mathbb{F}_{2}^{2 n}} V_{A_{1}^{2 n}+C} \otimes V_{A_{1}^{2 n}+C} \\
& \cong \bigoplus_{C \in \mathcal{Z}_{4 n}} V_{A_{1}^{4 n}+C}
\end{aligned}
$$

where

$$
\mathcal{Z}_{2 m}:=\left\{C=\left(c_{1}, \ldots, c_{2 m}\right) \in \mathbb{F}_{2}^{2 m} \mid c_{i}=c_{m+i} \text { for } 1 \leq i \leq m\right\}
$$

Observe that $\mathcal{Z}_{2 m}=\mathcal{D}_{2 m} \cup \mathcal{D}_{2 m}+[2]$ in the notation of (2.4). By applying Lemma 2.1 and noting that $D_{2 m} \cup D_{2 m}+[2] \cong \mathbb{Z}^{2 m}$ we obtain the following result.

Proposition 5.2. For $n$ a positive integer, the bulk of the diagonal $L_{1}\left(\mathfrak{s l}_{2}\right)^{\otimes 2 n}$ conformal field theory is isomorphic to $F(8 n)$ as a $L_{1}\left(\mathfrak{s l}_{2}\right)^{\otimes 2 n} \otimes L_{1}\left(\mathfrak{s l}_{2}\right)^{\otimes 2 n}$-module.

Proposition 5.2 furnishes bulk conformal field theory interpretations for the vertex operator superalgebras $F(8), F(16)$ and $F(24)$ in Theorem 3.1 .

It is instructive to consider the analogue of Proposition 5.2 where $L_{1}\left(\mathfrak{s l}_{2}\right)^{\otimes 2 n}$ is replaced by a simple current extension. Write $\left(1^{2 n}\right)$ as a shorthand for the "all ones" vector $(1,1, \ldots, 1) \in \mathbb{F}_{2}^{2 n}$. We will consider $V^{\prime} \cong V^{\prime \prime} \cong V_{L}$ where $L=A_{1}^{2 n} \cup$ $A_{1}^{2 n}+\left(1^{2 n}\right)$. Observe that the irreducible $V_{L}$-modules are the $V_{L+C}=V_{A_{1}^{2 n}+C} \oplus$ $V_{A_{1}^{2 n}+\left(1^{2 n}\right)+C}$ for $C \in \mathbb{F}_{2}^{2 n}$ with $\operatorname{wt}(C)=0 \bmod 2$. For simplicity assume that $n$ is even, so that $L$ is an even lattice and $V_{L}$ is a vertex operator algebra. Then for the bulk of the diagonal conformal field theory for $V_{L}$ we have

$$
\begin{aligned}
\mathcal{H}= & \bigoplus_{\substack{C \in \mathbb{F}_{2}^{2 n} \\
\mathrm{wt}(C)=0 \mathrm{mod} 2 \\
c_{2 n}=0}} V_{L+C} \otimes V_{L+C} \\
= & \bigoplus_{\substack{C \in \mathbb{F}_{2}^{2 n} \\
\mathrm{wt}(C)=0 \bmod 2}}\left(V_{A_{1}^{2 n}+C} \otimes V_{A_{1}^{2 n}+C} \oplus V_{A_{1}^{2 n}+\left(1^{2 n}\right)+C} \otimes V_{A_{1}^{2 n}+C}\right) .
\end{aligned}
$$

Comparing with (2.4) we see that

$$
\mathcal{H} \cong \bigoplus_{C \in \mathcal{D}_{4 n}^{+}} V_{A_{1}^{4 n}+C}
$$

where $\mathcal{D}_{4 n}^{+}:=\mathcal{D}_{4 n} \cup \mathcal{D}_{4 n}+[1]$. Since $D_{4 n}^{+}=D_{4 n} \cup D_{4 n}+[1]$ by definition, an application of Lemma 2.1 yields the following alternative interpretation for $V_{D_{4 n}^{+}}$as 
a potential bulk conformal field theory, at least for $n$ even.

Proposition 5.3. Let $n$ be an even positive integer and let $L=A_{1}^{2 n} \cup A_{1}^{2 n}+\left(1^{2 n}\right)$. Then the bulk diagonal conformal field theory associated to $V_{L}$ is isomorphic to $V_{D_{4 n}^{+}}$ as a $V_{L} \otimes V_{L}$-module.

Proposition 5.3 offers a bulk conformal field theory interpretation for $V_{E_{8}}$ distinct from that of Proposition 5.1 .

\subsection{Type D Superconformal Field Theory}

We now consider the diagonal superconformal field theory associated to $2 n$ free fermions. By the boson-fermion correspondence we have $F(2 n) \cong V_{D_{n}} \oplus V_{D_{n}+[2]}$, and $F(2 n)_{\mathrm{tw}} \cong V_{D_{n}+[1]} \oplus V_{D_{n}+[3]}$. So we have

$$
\mathcal{H}_{\mathrm{NS}-\mathrm{NS}}=F(2 n) \otimes F(2 n), \quad \mathcal{H}_{\mathrm{R}-\mathrm{R}}=F(2 n)_{\mathrm{tw}} \otimes F(2 n)_{\mathrm{tw}},
$$

in this case. This gives us a bulk superconformal field theory interpretation for $F(4 n)$ for $n$ a positive integer (cf. Proposition 5.2).

Proposition 5.4. Let $n$ be a positive integer. Then the NS-NS sector of the bulk diagonal superconformal field theory associated to $2 n$ free fermions is isomorphic to $F(4 n)$ as a $F(2 n) \otimes F(2 n)$-module, and the $R$ - $R$ sector is isomorphic to $F(4 n)_{\mathrm{tw}}$ as a canonically twisted $F(2 n) \otimes F(2 n)$-module.

Next we consider the diagonal superconformal field theory associated to the $D_{2 n}$ torus. In this case $V^{\prime}=V^{\prime \prime}=V_{D_{2 n}} \otimes F(2 n)$, so

$$
\mathcal{H}_{\mathrm{NS}-\mathrm{NS}}=\bigoplus_{i}\left(V_{D_{2 n}+[i]} \otimes F(2 n)\right) \otimes\left(V_{D_{2 n}+[i]} \otimes F(2 n)\right)
$$

as modules for $V^{\prime} \otimes V^{\prime \prime}$, and

$$
\mathcal{H}_{\mathrm{R}-\mathrm{R}}=\bigoplus_{i}\left(V_{D_{2 n}+[i]} \otimes F(2 n)_{\mathrm{tw}}\right) \otimes\left(V_{D_{2 n}+[i]} \otimes F(2 n)_{\mathrm{tw}}\right)
$$

as canonically twisted modules for $V^{\prime} \otimes V^{\prime \prime}$. Comparing this description with the decomposition of $D_{4 n}^{+}=D_{4 n} \cup D_{4 n}+$ [1] into cosets for $D_{2 n} \oplus D_{2 n}$ (cf. (5.2) ) we obtain the following identification.

Proposition 5.5. Let $n$ be a positive integer and set $V=V_{D_{2 n}} \otimes F(2 n)$. Then the NS-NS sector of the bulk diagonal superconformal field theory associated to the $D_{2 n}$ torus is isomorphic to $V_{D_{4 n}^{+}} \otimes F(4 n)$ as a $V \otimes V$-module, and the $R$ - $R$ sector is isomorphic to $V_{D_{4 n}^{+}} \otimes F(4 n)_{\mathrm{tw}}$ as a canonically twisted $V \otimes V$-module.

We also obtain our first example of a potential bulk superconformal field theory with superconformal structure. 
Theorem 5.6. Let $n$ be a positive integer. Then the vertex operator superalgebra $W=V_{D_{4 n}^{+}} \otimes F(4 n)$ is a potential bulk $N=(2,2)$ superconformal field theory in the sense of \$4 for $V^{\prime} \cong V^{\prime \prime} \cong V_{D_{2 n}} \otimes F(2 n)$, and the elliptic genus defined by this structure vanishes.

Proof. The modularity (4.21) and spectral flow symmetry (4.23) follow from Proposition 4.7. We may compute directly that the elliptic genus (4.25) vanishes, so it trivially satisfies (4.22).

Taking $n=2$ in Theorem 5.6 we obtain an interpretation for $V_{E_{8}} \otimes F(8)$ as the bulk superconformal field theory of the sigma model with $D_{4}$ torus as target. Volpato has observed [86] that supersymmetry preserving symmetry groups of sigma models with arbitrary 4-dimensional torus as target space embed in the Weyl group of $E_{8}$. The Weyl group of $E_{8}$ acts naturally on $V_{E_{8}} \otimes F(8)$. It appears that $V_{E_{8}} \otimes F(8)$ can play the analogous role for sigma models on 4-dimensional tori that $V_{D_{12}^{+}}$has been shown [34] to play for sigma models on K3 surfaces.

\subsection{Type A Superconformal Field Theory}

In principle we may consider superconformal field theories with $V^{\prime} \cong V^{\prime \prime} \cong V_{L}$ for $L=A_{1}^{2 n} \cup A_{1}^{2 n}+\left(1^{2 n}\right.$ ) also for $n$ odd (cf. Proposition 5.3). However, as we have explained in $\$ 2.4$, the case $n=3$ is distinguished by the presence of $N=4$ superconformal structure. This fact underpins a significant part of the analysis of [53], in which it is shown that the diagonal superconformal field theory with $V^{\prime} \cong$ $V^{\prime \prime} \cong V_{L}$ underlies a Kummer type $\mathrm{K} 3$ sigma model arising from the canonical $\mathbb{Z} / 2 \mathbb{Z}$ orbifold of the $D_{4}$ torus. This is the tetrahedral K3 sigma model in [80]. We discuss this sigma model from the point of view of $A_{1}^{6}$ now. Although the motivation of [53] is somewhat different, their detailed analysis precedes, and may serve to flesh out the discussion we offer here.

So let $L=A_{1}^{6} \cup A_{1}^{6}+\left(1^{6}\right)$ in this section. Directly applying the observations preceding Proposition 5.3 with $n=3$ we see that the NS-NS sector of the diagonal superconformal field theory associated to $V_{L}$ satisfies $\mathcal{H}_{\mathrm{NS}-\mathrm{NS}} \cong V_{D_{12}} \oplus V_{D_{12}+[1]}=$ $V_{D_{12}^{+}}$as a $V_{L} \otimes V_{L}$-module. Noting that the irreducible canonically twisted $V_{L}$-modules are the $V_{L+C}$ with $C \in \mathbb{F}_{2}^{6}$ and $\operatorname{wt}(C)=1 \bmod 2$ we find that $\mathcal{H}_{\mathrm{R}-\mathrm{R}} \cong V_{D_{12}+[2]} \oplus$ $V_{D_{12}+[3]}$ as a canonically twisted $V_{L} \otimes V_{L}$-module. The diagonal superconformal field theory for $V_{L}$ underlies the tetrahedal K3 sigma model according to [53], so we have the following super-analogue of Proposition 5.3 for $n=3$.

Proposition 5.7. For $L=A_{1}^{6} \cup A_{1}^{6}+\left(1^{6}\right)$, the $N S$ - NS sector of the tetrahedral K3 sigma model is isomorphic to $V_{D_{12}^{+}}$as a $V_{L} \otimes V_{L}$-module, and the $R$ - $R$ sector of the tetrahedral K3 sigma model is isomorphic to $V_{D_{12}+[2]} \oplus V_{D_{12}+[3]}$ as a canonicallytwisted $V_{L} \otimes V_{L}$-module. 
Now let us consider superconformal structure. As explained in 92.4 the vertex operator superalgebra $V_{L} \cong V_{A_{1}^{6}} \oplus V_{A_{1}^{6}+\left(1^{6}\right)}$ contains a copy of the vacuum module for the small $N=4$ superconformal algebra at $c=6$. As in 2.4 we choose the first copy of $L_{1}\left(\mathfrak{s l}_{2}\right)$ in $L_{1}\left(\mathfrak{s l}_{2}\right)^{\otimes 6}<V_{L}$ to generate the affine $\mathfrak{s l}_{2}$ sub algebra. Then spectral flow corresponds to fusion with $V_{L+C} \otimes V_{L+C}$ where $C=\left(10^{5}\right)$. In terms of $V_{D_{12}^{+}}$this is the same as fusion with $V_{D_{12}+[2]}$ by force of (2.4). Thus spectral flow interchanges the NS-NS and R-R sectors. An explicit calculation, such as is carried out in $\S \mathrm{D} .3$ of [53], verifies that $E_{W}(u, \tau)$ is a weak Jacobi form of weight 0 and index 1 such that $E_{W}(0, \tau)=24$. Thus we have the following.

Theorem 5.8. The vertex operator superalgebra $V_{D_{12}^{+}}$is a potential bulk $N=(4,4)$ superconformal field theory in the sense of \$4 for $V^{\prime} \cong V^{\prime \prime} \cong V_{L}$, and the elliptic genus defined by this structure is the K3 elliptic genus.

Note that $V_{D_{12}^{+}}$serves as the moonshine module for Conway's group [30, 33], and is precisely the vertex operator superalgebra that is used to attach weak Jacobi forms with level to supersymmetry preserving symmetries of K3 sigma models in [34. Results equivalent to Proposition 5.7 and Theorem 5.8 have been obtained independently in 82$]$.

\subsection{Gepner Type Superconformal Field Theory}

We now present a superconformal field theory interpretation for $V_{D_{12}^{+}}$of a different nature. As mentioned in $\$ 2.1$, the lattice vertex operator superalgebra $V_{\sqrt{3} \mathbb{Z}}$ realizes the vacuum module of the $N=2$ superconformal algebra at $c=1$. In this section we set $K=\sqrt{3} \mathbb{Z}^{6}$. Thus $V_{K}$ contains the vacuum module of the $N=2$ superconformal algebra at $c=6$. We will show that $W=V_{D_{12}^{+}}$is a quasi potential bulk $N=(2,2)$ superconformal field theory (cf. Definition 4.6) for $V^{\prime} \cong V^{\prime \prime} \cong V_{K}$. It will develop that $W$ is closely related to the Gepner model of type $(1)^{6}$, which is a superconformal field theory that also has $V^{\prime} \cong V^{\prime \prime} \cong V_{K}$.

Recall from 92.1 that the lattice $\sqrt{3} \mathbb{Z}^{12} \cong K \oplus K$ embeds in $D_{12}^{+}$. Using such an embedding we may fix commuting sub vertex operator superalgebras $V^{\prime}, V^{\prime \prime}<W$ such that $V^{\prime} \cong V^{\prime \prime} \cong V_{K}$. Since the discriminant group of $K \oplus K$ is $\mathbb{F}_{3}^{12}$ it is natural to use ternary codewords of length 12 to describe the irreducible $V_{K} \otimes V_{K}$-modules. According to Lemma 2.2 the $V_{K} \otimes V_{K}$-modules that appear in $W$ are indexed by the codewords in a copy $\mathcal{G}_{12}^{+}$of the ternary Golay code. To make this more concrete let us assume that $\left(+{ }^{6}-^{6}\right.$ ) is a word in $\mathcal{G}_{12}^{+}$(if not then permute the coordinates so as to make this true), and define $C^{\prime}, C^{\prime \prime} \in \mathbb{F}_{3}^{6}$ for $C \in \mathcal{G}_{12}^{+}$by setting $C^{\prime}=\left(c_{1}, \ldots, c_{6}\right)$ and $C^{\prime \prime}=\left(c_{7}, \ldots, c_{12}\right)$ when $C=\left(c_{1}, \ldots, c_{12}\right)$. Then we have

$$
W=\bigoplus_{C \in \mathcal{G}_{12}^{+}} V_{K+C^{\prime}} \otimes V_{K+C^{\prime \prime}}
$$


as modules for $V_{K} \otimes V_{K}$. Our main result in this section is the following.

Theorem 5.9. The vertex operator superalgebra $V_{D_{12}^{+}}$is a quasi potential bulk $N=$ $(2,2)$ superconformal field theory in the sense of $\$$, for $V^{\prime} \cong V^{\prime \prime} \cong V_{K}$, and the elliptic genus defined by this structure is the K3 elliptic genus.

Proof. Let $w$ be the marked complete weight enumerator of $\mathcal{G}_{12}^{+}$for the marking $C \mapsto\left(C^{\prime}, C^{\prime \prime}\right)$. That is, define $w$ to be the 6 -variate polynomial

$$
w\left(X^{\prime}, Y^{\prime}, Z^{\prime}, X^{\prime \prime}, Y^{\prime \prime}, Z^{\prime \prime}\right):=\sum_{C \in \mathcal{G}_{12}^{+}} w_{C^{\prime}}\left(X^{\prime}, Y^{\prime}, Z^{\prime}\right) w_{C^{\prime \prime}}\left(X^{\prime \prime}, Y^{\prime \prime}, Z^{\prime \prime}\right)
$$

where $w_{C}(X, Y, Z)$, for $C \in \mathbb{F}_{3}^{n}$, is defined by $w_{C}(X, Y, Z):=X^{a_{0}} Y^{a_{+}} Z^{a_{-}}$in case $C$ has $a_{0}$ entries equal to 0 , and $a_{ \pm}$entries equal to \pm 1 . Then the functions $\widehat{\mathrm{ch}}^{ \pm}[W]\left(u^{\prime}, \tau^{\prime}, u^{\prime \prime}, \tau^{\prime \prime}\right)$ and $\widehat{c h}^{ \pm}\left[W_{\mathrm{tw}}\right]\left(u^{\prime}, \tau^{\prime}, u^{\prime \prime}, \tau^{\prime \prime}\right)$ (cf. (4.19) $)$ are obtained by substituting characters of suitable irreducible modules for the $N=2$ superconformal algebra at $c=1$. These characters can be expressed in terms of classical theta functions and the Dedekind eta function (cf. [74]) so they are invariant for the action of some finite index subgroup of $S L_{2}(\mathbb{Z})$. So $Z_{W}$ (cf. (4.5) ) is invariant for some finite index subgroup of $S L_{2}(\mathbb{Z})$. Also, the $N=2$ characters satisfy spectral flow symmetry, so the same is true for $W$.

It remains to examine the elliptic genus $E_{W}$ (cf. (4.25) $)$ of $W$. For this define

$$
f_{s}(u, \tau):=\eta(\tau)^{-1} \sum_{k \in \mathbb{Z}}\left(e^{\pi i} z\right)^{k+\frac{s}{6}} q^{\frac{3}{2}\left(k+\frac{s}{6}\right)^{2}}
$$

which is a character for the $N=2$ superconformal algebra at $c=1$ when $s \in \mathbb{Z}$. Note that $f_{s}$ depends only on $s \bmod 6$, we have $f_{s}(0, \tau)=e^{ \pm \frac{\pi i}{6}}$ when $s= \pm 1 \bmod 6$, and $f_{s}(0, \tau)$ vanishes identically when $s=3 \bmod 6$. Because of this we have

$$
E_{W}(u, \tau)=w\left(f_{3}(u, \tau), f_{1}(u, \tau), f_{-1}(u, \tau), 0, e^{\frac{\pi i}{6}}, e^{-\frac{\pi i}{6}}\right) .
$$

Under our hypotheses on $\mathcal{G}_{12}^{+}$the marked complete weight enumerator is given by

$$
\begin{aligned}
w\left(X^{\prime}, Y^{\prime}, Z^{\prime},\right. & \left.X^{\prime \prime}, Y^{\prime \prime}, Z^{\prime \prime}\right)=\left(X^{\prime 6}+Y^{\prime 6}+Z^{\prime 6}\right)\left(X^{\prime \prime}{ }^{6}+Y^{\prime \prime} 6+Z^{\prime \prime 6}\right) \\
& +90\left(X^{\prime 4} Y^{\prime} Z^{\prime}+X^{\prime} Y^{\prime 4} Z^{\prime}+X^{\prime} Y^{\prime} Z^{\prime 4}\right) X^{\prime \prime 2} Y^{\prime \prime 2} Z^{\prime \prime 2} \\
& +20\left(X^{\prime 3} Y^{\prime 3}+X^{\prime 3} Z^{\prime 3}+Y^{\prime 3} Z^{\prime 3}\right)\left(X^{\prime \prime 3} Y^{\prime \prime 3}+X^{\prime \prime 3} Z^{\prime \prime 3}+Y^{\prime \prime 3} Z^{\prime \prime 3}\right) \\
& +90 X^{\prime 2} Y^{\prime 2} Z^{\prime 2}\left(X^{\prime \prime} Y^{\prime \prime} Z^{\prime \prime}+X^{\prime \prime} Y^{\prime \prime} Z^{\prime \prime}+X^{\prime \prime} Y^{\prime \prime} Z^{\prime \prime 4}\right) .
\end{aligned}
$$

So we have

$$
E_{W}=-2\left(f_{3}{ }^{6}+f_{1}{ }^{6}+f_{-1}{ }^{6}\right)+20\left(f_{1}^{3} f_{-1}{ }^{3}+f_{3}{ }^{3} f_{1}{ }^{3}+f_{-1}{ }^{3} f_{3}{ }^{3}\right) .
$$


We may check directly using (5.12) that this expression for $E_{W}$ is a weak Jacobi form of weight 0 and index 1 . Substituting $u=0$ in (5.15) we obtain $E_{W}(0, \tau)=$ $-2((-1)+(-1))+20(1)=24$. So $E_{W}$ is indeed the K3 elliptic genus. This completes the proof.

It is interesting to compare the quasi potential bulk superconformal field theory structure (5.10) to the superconformal field theory underlying the Gepner model of type $(1)^{6}$, which realizes a K3 sigma model and also has $V^{\prime} \cong V^{\prime \prime} \cong V_{K}$. To describe the bulk define a ternary code $\mathcal{U}$ of length 6 by setting

$$
\mathcal{U}:=\left\{C=\left(c_{1}, \ldots, c_{6}\right) \in \mathbb{F}_{3}^{6} \mid \sum_{i} c_{i}=0\right\} .
$$

Then, according to [51] for example, the NS-NS sector is $\mathcal{H}_{\mathrm{NS}-\mathrm{NS}}=\bigoplus_{a \in \mathbb{F}_{3}} \mathcal{H}_{\mathrm{NS}-\mathrm{NS}}^{a}$ where

$$
\mathcal{H}_{\mathrm{NS}-\mathrm{NS}}^{a}:=\bigoplus_{C \in \mathcal{U}} V_{K+C+\left(a^{6}\right)} \otimes V_{K+C-\left(a^{6}\right)}
$$

as a module for $V_{K} \otimes V_{K}$. Since there are $V_{K} \otimes V_{K}$-modules in (5.17) whose corresponding codewords in $\mathbb{F}_{3}^{12}$ have fewer than 6 non-zero entries, the $V_{K} \otimes V_{K}$-module structure on $W$ in Theorem 5.9 does not identify it with the superconformal field theory underlying the Gepner model $(1)^{6}$.

However, $W$ and the $(1)^{6}$ model have closely related symmetry. To see this let $G^{N=2}$ be the group of symmetries of the bulk superconformal field theory of the $(1)^{6}$ model that fix the states of the left and right moving $N=2$ superconformal algebras at $c=6$. According to [51] this group is a split extension of $S_{6}$ by $(\mathbb{Z} / 3 \mathbb{Z})^{6}$. The subgroup of the automorphism group of $\mathcal{G}_{12}^{+}$that stabilizes the splitting $C \mapsto\left(C^{\prime}, C^{\prime \prime}\right)$ (cf. (5.10) ) is another copy of $S_{6}$. From this it follows that $G^{N=2}$ also acts on $W$, preserving the states of the two commuting $N=2$ superconformal algebras at $c=6$ in $V_{K} \otimes V_{K}$.

Now let $G^{N=4}$ be the subgroup of $G^{N=2}$ that preserves the left and right moving copies of the small $N=4$ superconformal algebra. According to the results of 34] the actions of $G^{N=4}$ on $W$ and the bulk of the $(1)^{6}$ model give the same equivariant elliptic genera. We hope that the notion of quasi potential bulk superconformal field theory will help to identify an abstract mechanism that explains this coincidence.

There are a number of $c=6$ combinations of minimal $N=2$ models $V$ such that $V \otimes V$ embeds in $V_{D_{12}^{+}}$. Does every such embedding give rise to a quasi potential $N=(2,2)$ superconformal field theory with a close relationship to the corresponding Gepner model? It would be valuable to have a complete classification of these embeddings. 


\section{Acknowledgements}

The authors are grateful to Anne Taormina and Katrin Wendland for correspondence regarding their recent manuscript [82]. T.C. appreciates correspondence with Geoff Mason, and gratefully acknowledges support from the Natural Sciences and Engineering Research Council of Canada (RES0020460). J.D. thanks Anne Taormina and Katrin Wendland for discussions on related topics, and gratefully acknowledges support from the U.S. National Science Foundation (DMS 1203162, DMS 1601306), and the Simons Foundation (\#316779).

\section{References}

[1] A. Ali, Classification of two-dimensional $N=4$ superconformal symmetries, hep-th/9906096.

[2] D. Ben-Zvi, R. Heluani and M. Szczesny, Supersymmetry of the chiral de Rham complex Compos. Math. 144 (2008), no. 2, 503-521.

[3] R. E. Borcherds, Monstrous moonshine and monstrous Lie superalgebras, Invent. Math. 109, No.2 (1992), 405-444.

[4] L A. Borisov, Vertex algebras and mirror symmetry, Comm. Math. Phys. 215 (2001), no. 3, 517-557.

[5] L A. Borisov and A. Libgober, Elliptic genera of toric varieties and applications to mirror symmetry, Invent. Math. 140 (2000), no. 2, 453-485.

[6] M. C. N. Cheng, K3 surfaces, $N=4$ dyons and the Mathieu group $M_{24}$, Commun. Number Theory Phys. 4 (2010), no. 4, 623-657.

[7] M. Cheng, X. Dong, J. Duncan, S. Harrison, S. Kachru, and T. Wrase, Mock Modular Mathieu Moonshine Modules, Res. Math. Sci. (2015) 213

[8] M. C. N. Cheng, J. F. R. Duncan, S. M. Harrison and S. Kachru, Equivariant K3 Invariants, (To appear in Commun. Number Theory Phys.) arXiv:1508.02047.

[9] M. C. N. Cheng, J. F. R. Duncan, and J. A. Harvey. Umbral Moonshine, Commun. Number Theory Phys., 8(2):101-242, 2014.

[10] M. C. N. Cheng, J. F. R. Duncan, and J. A. Harvey. Umbral Moonshine and the Niemeier Lattices, Res. Math. Sci., 1(3):1-81, 2014.

[11] M. C. N. Cheng, S. M. Harrison, S. Kachru and D. Whalen, Exceptional Algebra and Sporadic Groups at c=12, arXiv:1503.07219. 
[12] M. C. N. Cheng, S. M. Harrison, R. Volpato and M. Zimet, K3 String Theory, Lattices and Moonshine, arXiv:1612.04404.

[13] J. H. Conway, A perfect group of order 8,315, 553, 613, 086, 720, 000 and the sporadic simple groups, Proc. Nat. Acad. Sci. U.S.A. 61 (1968), 398-400.

[14] J. H. Conway, A group of order 8, 315, 553, 613, 086, 720, 000, Bull. London Math. Soc. 1 (1969), 79-88.

[15] J. H. Conway and S. P. Norton, Monstrous moonshine, Bull. London Math. Soc. 11 (1979), no. 3, 308-339.

[16] J. H. Conway and N. J. A. Sloane, Sphere packings, lattices and groups, Springer-Verlag, 1999.

[17] T. Creutzig and G. Höhn, Mathieu Moonshine and the Geometry of K3 Surfaces, Commun. Num. Theor. Phys. 8, 2 (2014) 295-328.

[18] T. Creutzig, S. Kanade and A. Linshaw, Simple current extensions beyond semi-simplicity, arXiv:1511.08754.

[19] T. Creutzig, S. Kanade and R. McRae, Tensor categories for vertex operator algebra extensions, in preparation.

[20] T. Creutzig and D. Ridout, Modular Data and Verlinde Formulae for Fractional Level WZW Models II, Nucl. Phys. B 875 (2013) 423.

[21] P. Di Francesco and S. Yankielowicz, Ramond sector characters and $N=2$ Landau-Ginzburg models Nuclear Phys. B 409 (1993), no. 1, 186-210.

[22] C. Dong, H. Li and G. Mason, Twisted representations of vertex operator algebras Math. Ann. 310 (1998), no. 3, 571-600.

[23] C. Dong, H. Li and G. Mason, Modular invariance of trace functions in orbifold theory and generalized Moonshine, Communications in Mathematical Physics 214 1-56, 2000.

[24] C. Dong, H. Li, G. Mason, and P. S. Montague, The radical of a vertex operator algebra, In The Monster and Lie algebras (Columbus, OH, 1996), volume 7 of Ohio State Univ. Math. Res. Inst. Publ., pages 17-25. de Gruyter, Berlin, 1998.

[25] C. Dong and G. Mason. Nonabelian orbifolds and the boson-fermion correspondence, Comm. Math. Phys., 163(3):523-559, 1994.

[26] C. Dong and G. Mason. Rational vertex operator algebras and the effective central charge, Int. Math. Res. Not., (56):2989-3008, 2004. 
[27] C. Dong and G. Mason. Integrability of $C_{2}$-cofinite vertex operator algebras, Int. Math. Res. Not., pages Art. ID 80468, 15, 2006.

[28] C. Dong and Z. Zhao. Modularity in Orbifold Theory for Vertex Operator Superalgebras, Commun. Math. Phys. 260, 227-256 (2005).

[29] C. Dong and Z. Zhao. Modularity of trace functions in orbifold theory for $\mathbb{Z}$ graded vertex operator superalgebras, Moonshine: the first quarter century and beyond, London Math. Soc. Lecture Note Ser. 372, Cambridge Univ. Press, Cambridge, 2010.

[30] J. F. Duncan. Super-Moonshine for Conway's largest sporadic group, Duke Math. J., 139(2):255-315, 2007.

[31] J. F. R. Duncan, M. J. Griffin, and K. Ono, Moonshine, Res. Math. Sci., $2(11), 2015$.

[32] J. F. R. Duncan, M. J. Griffin, and K. Ono, Proof of the Umbral Moonshine Conjecture, Res. Math. Sci., 2(26), March 2015.

[33] J. Duncan and S. Mack-Crane The Moonshine Module for Conway's Group, Forum Math. Sigma 3 (2015), arxiv:1409.4124.

[34] J. Duncan and S. Mack-Crane Derived Equivalences of K3 Surfaces and Twined Elliptic Genera, Res. Math. Sci. (2016) 3:1. arXiv:1506.06198.

[35] J. F. R. Duncan, M. H. Mertens and K. Ono, O'Nan Moonshine and Arithmetic, arXiv:1702.03516.

[36] T. Eguchi and K. Hikami, Note on Twisted Elliptic Genus of K3 Surface, Phys. Lett. B694 (2011), 446-455.

[37] T. Eguchi, H. Ooguri and Y. Tachikawa. Notes on the K3 Surface and the Mathieu group $M_{24}$, Exper. Math., 20:91-96, 2011.

[38] T. Eguchi, H. Ooguri, A. Taormina and S.-K. Yang, Superconformal Algebras and String Compactification on Manifolds with $S U(N)$ Holonomy, Nucl. Phys. B315 (1989) 193-221.

[39] T. Eguchi and A. Taormina, Unitary representations of the $N=4$ superconformal algebra, Phys. Lett. B196, 75-81 (1987).

[40] T. Eguchi and A. Taormina, Character formulas for the $N=4$ superconformal algebra, Phys. Lett. B200, 315-322 (1988).

[41] T. Eguchi and A. Taormina, On the unitary representations of $N=2$ and $N=4$ superconformal algebras, Phys. Lett. 210, 125-132 (1988). 
[42] E. Frenkel and D. Ben-Zvi, Vertex algebras and algebraic curves, vol. 88 of Mathematical Surveys and Monographs, American Mathematical Society, Providence, RI, second ed., 2004.

[43] I. B. Frenkel. Two constructions of affine Lie algebra representations and boson-fermion correspondence in quantum field theory, J. Funct. Anal., 44(3):259-327, 1981.

[44] I. Frenkel, J. Lepowsky and A. Meurman, A natural representation of the Fischer-Griess Monster with the modular function $J$ as character, Proc. Nat. Acad. Sci. U.S.A. 81 (1984), no. 10, Phys. Sci., 3256-3260.

[45] I. Frenkel, J. Lepowsky and A. Meurman, A moonshine module for the Monster, Vertex operators in mathematics and physics (Berkeley, Calif., 1983), Math. Sci. Res. Inst. Publ., vol. 3, Springer, New York, 1985, pp. 231273.

[46] I. Frenkel, J. Lepowsky and A. Meurman, Vertex operator algebras and the Monster, Pure and Applied Mathematics, vol. 134, Academic Press Inc., Boston, MA, 1988.

[47] J. Fuchs, I. Runkel and C. Schweigert, TFT construction of RCFT correlators I: Partition functions, Nucl. Phys. B 646, 353-497 (2002).

[48] M. R. Gaberdiel, An Introduction to Conformal Field Theory, Rept. Prog. Phys. (2000) 63, 607-667.

[49] M. Gaberdiel, S. Hohenegger, and R. Volpato, Mathieu twining characters for K3, JHEP 1009 (2010), 058, 19 pages.

[50] M. Gaberdiel, S. Hohenegger, and R. Volpato, Mathieu Moonshine in the elliptic genus of K3, JHEP 1010 (2010), 062.

[51] M. R. Gaberdiel, S. Hohenegger, and R. Volpato, Symmetries of K3 sigma models, Commun. Number Theory Phys., 6(1):1-50, 2012.

[52] M. R. Gaberdiel, C. A. Keller, and P. Hynek, Mathieu Moonshine and Symmetry Surfing, arXiv:1609.09302.

[53] M. R. Gaberdiel, A. Taormina, R. Volpato and K. Wendland, A K3 sigma model with $\mathbb{Z}_{2}^{8}: \mathbb{M}_{20}$ symmetry, JHEP 1402 (2014) 022.

[54] T. Gannon, Much ado about Mathieu, Adv. Math. 301 (2016), 322-358, arXiv:1211.5531.

[55] P. Goddard and A. Schwimmer. Factoring Out Free Fermions and Superconformal Algebras, Phys. Lett. B 214(2):209-214, 1988. 
[56] M. J. Griffin and M. H. Mertens, A proof of the Thompson Moonshine Conjecture, Res. Math. Sci. 3 (2016), Art. 36, 32 pp.

[57] J. Han and C. Ai, Three equivalent rationalities of vertex operator superalgebras J. Math. Phys. 56, 111701 (2015).

[58] J. A. Harvey and B. C. Rayhaun, Traces of Singular Moduli and Moonshine for the Thompson Group, Commun. Number Theory Phys. 10 (2016), no. $1,23-62$.

[59] R. Heluani, Supersymmetry of the chiral de Rham complex. II. Commuting sectors Int. Math. Res. Not. IMRN 6 (2009), 953-987.

[60] F. Hirzebruch, Topological methods in algebraic geometry. Third enlarged edition. New appendix and translation from the second German edition by R. L. E. Schwarzenberger, with an additional section by A. Borel. Die Grundlehren der Mathematischen Wissenschaften, Band 131 Springer- Verlag New York, Inc., New York 1966 x+232 pp.

[61] G. Höhn, Komplexe elliptische Geschlechter und $S^{1}$-äquivariante Kobordismustheorie, Diploma thesis, Bonn 1991, arXiv:math/0405232.

[62] G. Höhn. Selbstduale vertexoperatorsuperalgebren und das babymonster (selfdual vertex operator super algebras and the baby monster), Bonner Mathematische Schriften, Vol., 286,:1-85, Bonn 1996, June 2007.

[63] Y.-Z. Huang, Vertex operator algebras and conformal field theory, Internat. J. Modern Phys. A 7 (1992), no. 10, 2109-2151.

[64] V. Kac. Vertex algebras for beginners, volume 10 of University Lecture Series, American Mathematical Society, Providence, RI, second edition, 1998.

[65] A. Kapustin and D. Orlov, Vertex algebras, mirror symmetry, and D-branes: the case of complex tori, Comm. Math. Phys. 233 (2003), no. 1, 79-136.

[66] S. H. Katz, A. Klemm and R. Pandharipande, On the motivic stable pairs invariants of K3 surfaces, K3 surfaces and their moduli, Birkhäuser/Springer, Progr. Math. 315 (2016), 111-146.

[67] S. H. Katz, A. Klemm and C. Vafa, M theory, topological strings and spinning black holes, Adv. Theor. Math. Phys. 3, 1445 (1999) [hep-th/9910181].

[68] M. Krauel and M. Miyamoto, A modular invariance property of multivariable trace functions for regular vertex operator algebras, arXiv:1501.00124. 
[69] I. M. Krichever, Generalized elliptic genera and Baker-Akhiezer functions, (Russian) Mat. Zametki 47 (1990), 34-45; translation in Math. Notes 47 (1990), 132-142.

[70] J. Lepowsky and H. Li. Introduction to vertex operator algebras and their representations, volume 227 of Progress in Mathematics, Birkhäuser Boston Inc., Boston, MA, 2004.

[71] W. Nahm and K. Wendland, A Hiker's Guide to K3 - Aspects of $N=(4,4)$ Superconformal Field Theory with central charge $c=6$, Comm. Math. Phys. 216 (2001), 85-138.

[72] R. Pandharipande and R. P. Thomas, The Katz-Klemm-Vafa conjecture for K3 surfaces, Forum Math. Pi 4 (2016).

[73] R. A. Rankin. Modular forms and functions, Cambridge University Press, Cambridge, 1977.

[74] F. Ravanini and S.-K. Yang, Modular invariance in $N=2$ superconformal field theories, Phys. Lett. B. 195(2) 202-208, 1987.

[75] B. Song, The global sections of the chiral de Rham complex on a Kummer surface, Int. Math. Res. Not. IMRN 14 (2016), 4271-4296, arXiv:1312.7386.

[76] B. Song, Vector bundles induced from jet schemes, arXiv:1609.03688.

[77] H. Tamanoi, Symmetric invariant pairings in vertex operator super algebras and Grammians. Journal of Pure and Applied Algebra 140 (1999) 149-189.

[78] H. Tamanoi, Elliptic genera and vertex operator super-algebras, Lecture Notes in Mathematics, 1704, Springer-Verlag, 1999.

[79] A. Taormina and K. Wendland, Symmetry-surfing the moduli space of Kummer K3s String-Math 2012, Proc. Sympos. Pure Math. 90, Amer. Math. Soc., Providence, RI, 2015.

[80] A. Taormina and K. Wendland, The overarching finite symmetry group of Kummer surfaces in the Mathieu group $M_{24}$, JHEP 08 (2013) 125.

[81] A. Taormina and K. Wendland, A twist in the $M_{24}$ Moonshine story Confluentes Math. 7 (2015), no. 1, 83-113.

[82] A. Taormina and K. Wendland, The Conway Moonshine Module is a Reflected K3 Theory, arXiv preprint.

[83] J. G. Thompson, Finite groups and modular functions, Bull. London Math. Soc. 11 (1979), no. 3, 347-351. 
[84] J. G. Thompson, Some numerology between the Fischer-Griess Monster and the elliptic modular function, Bull. London Math. Soc. 11 (1979), no. 3, $352-353$.

[85] J. Van Ekeren, Modular invariance for twisted modules over a vertex operator superalgebra, Comm. Math. Phys. 322 (2013), no. 2, 333-371.

[86] R. Volpato, On symmetries of $\mathcal{N}=(4,4)$ sigma models on $T^{4}$, JHEP 1408 (2014) 094.

[87] K. Wendland, Snapshots of conformal field theory, Mathematical aspects of quantum field theories, Math. Phys. Stud., Springer, Cham, 2015.

[88] E. Witten, On the Landau-Ginzburg description of $N=2$ minimal models Internat. J. Modern Phys. A, 9 (1994), no. 27, 4783-4800.

[89] Y. Zhu, Modular invariance of characters of vertex operator algebras, J. Amer. Math. Soc. 9 (1996) 237-302. 